AIIL- $-8 \div-62$

ANL-84-62

DEE5 002710

\begin{abstract}
ARGONNE NATIONAL LABORATOFY
9700 South Cass Avenue

Argonne, Illinois 60439
\end{abstract}

MIXING, CHEMICAL REACTION AND FLOW FIELD DEVELOPMENT IN DUCTED ROCKETS

by

S. P. Vanka, R. R. Crafg, * and F. D. Stull*

Components Technology Division

September 1984

Work Performed at Argonne National Laboratory under Interagency Agreement FY 145583N0606

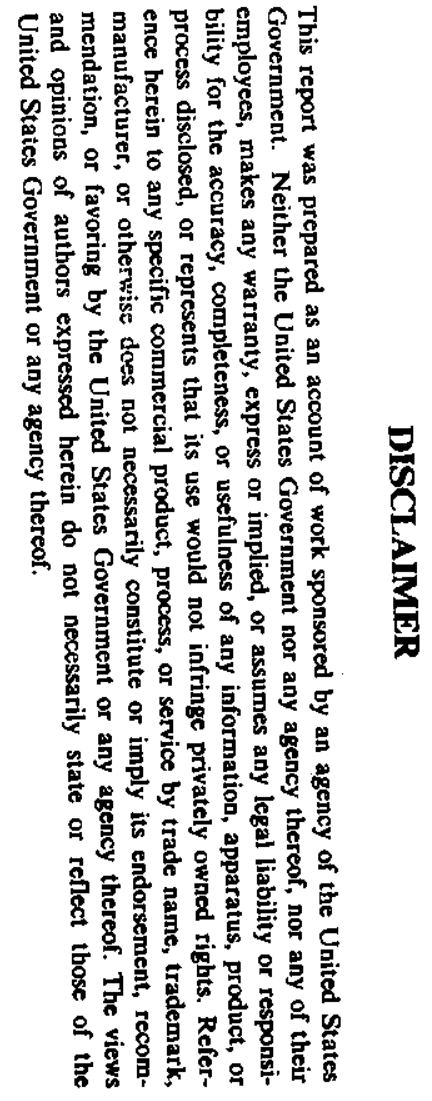

*Ramjet Technology Division, WPAFB, Oh1o 


\section{TABLE OF CONTENTS}

Page

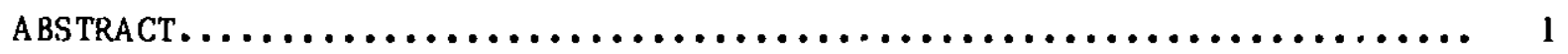

1. INTRODUCTION.................................... 1

2. Governing EqUATIONS............................... 4

3. SOLUTION ALGORITHM............................. 7

4. CALCULATED Flow AND SCALAR FIELDS $\ldots \ldots \ldots \ldots \ldots \ldots \ldots \ldots \ldots \ldots \ldots \ldots \ldots$

4.1 Computational Aspects........................... 8

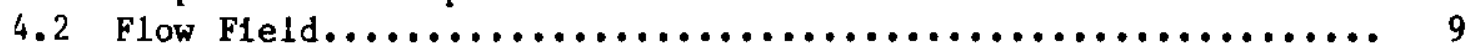

4.3 Temperature and Fuel Fraction Contours................. 10

5. INFLUENCE OF GEOMETRICAL PARAMETERS $\ldots \ldots \ldots \ldots \ldots \ldots \ldots \ldots \ldots \ldots \ldots \ldots$

5.1 Ef fect of Dome Height........................... 12

5.2 Effect of Side Arm Angle......................... 13

5.3 Effect of Injector Location........................ 14

6. $\quad$ SUMMARY......................................... 14

ACKNOWLEDGMENTS .................................... 42

REFERENCES.......................................... 42 


\section{FIGURES}

F1gure

Page

1 Ducted Rocket Combustor Configuration................. 16

2 Cross-Stream Flow Patterns for Base Configuration........... 17

3 Flow Patterns in Azimuthal Planes for Base Configuration...... 19

4 Cross-stream Temperature Distribution for Base Configuration... 21

5 Cross-stream Fuel Fraction Distribution for Base

Configuration.................................. 23

6 Axial Varfation of Average Fuel Fraction for Base

Configuration................................... 25

7 Axial Variation of Combustion Effictency for Base

Configuration.................................. 26

8 Flow Patterns in Selected Azimuthal Planes for 0.0 m Dome Height........................................ 27

9 Cross-stream Temperature Distributions for $0.0 \mathrm{~m}$ Dome Height... 29

10 Flow Patterns in Selected Azimuthal Planes for $0.116 \mathrm{~m}$ Dome Height........................................ 30

11 Flow Patterns in Selected Azimuthal Planes for $30^{\circ}$ Side-arm Angle.......................................... 31

12 Flow Patterns in Selected Azimuthal Planes for $60^{\circ}$ Side-arm Angle......................................... 32

13 Axial Variation of Average Fuel Fraction for Different Side-arm Angles................................... 33

14 Axial Variation of Average Combustion Ef ficlency for Different Side-arm Angles.................................. 34

15 Flow Patterns in Selected Azimuthal Plans for Concentric Fuel Injection..................................... 35

16 Cross-stream Temperature Distributions for Concentric Fue 1 Injection. .................................... 36

17 Axtal Vartation of Average Fuel Fraction for Concentric Fuel Injectton.................................... 38

18 Axial Variation of Average Combustion Efficiency for Concentric Fuel Injection................................... 


\section{TABIES}

Tab1e

Page

1 Exchange Coefficients and Additional Source Terms............ 40

$2 \quad$ Fuel Properties Used................................. 41

3 Conditions for Base Calculations....................... 41 


\section{NOMENCLATURE}

$a, b, c$ Parameters in the relation for specific heats

$A_{p}, A_{n} \quad$ Coefficients in the finite difference equations

$\mathrm{C}_{\mathrm{p}}$

$\mathrm{C}_{1}, \mathrm{C}_{2}, \mathrm{C}_{\mu}$ Constants in the turbulence model

$\mathrm{C}_{\mathrm{g} 1}, \mathrm{C}_{\mathrm{g} 2}$ Constants in the concentration fluctuation equation

f

$\mathrm{H}_{\mathrm{fu}}$

h

1

k

M

M Molecular weight

m Mass fraction

P Production of kinetic energy of turbulence

p Pressure

r Radial coordinate

R Gas constant

S Source term

$s^{u}, s^{p} \quad$ Comp nents of source term, $S=s^{\mathbf{u}}+s^{p}$

T Temperature

$\mathrm{u}, \mathrm{v}, \mathrm{w} \quad$ Velocity components in $\mathrm{x}, \mathrm{r}, \theta$ directions, respectively

\section{Greek Letters}

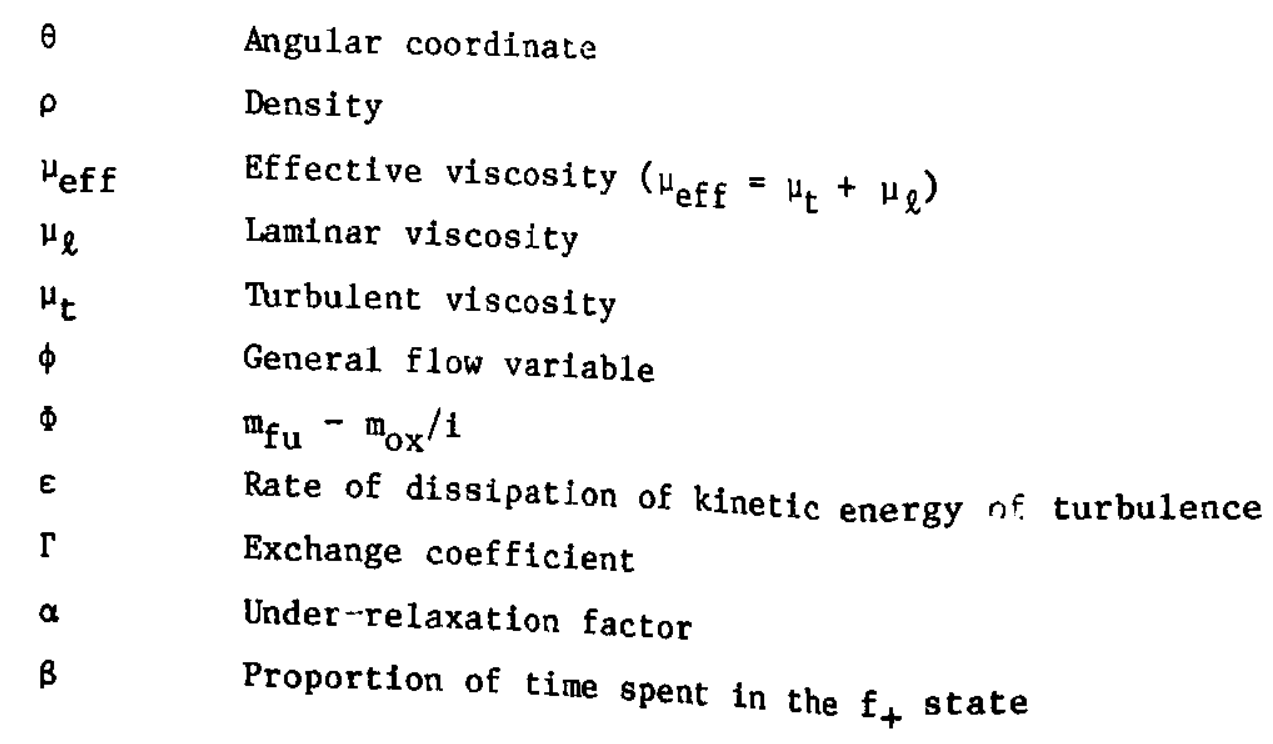

\section{Subscripts}

A

Air Stream

F Fue1 stream

fu Fue1

ox Oxidant

pr Product

$k \quad K i n e t i c$ energy of turbulence

$\varepsilon \quad$ Rate of Dissipation of $\mathrm{k}$

,+- Positive and negative sides of fluctuation 


\title{
MIXING, CHEMICAL REACTION AND FLON FIELD DEVELOPMENT IN DUCTED ROCKETS
}

by S. P. Vanka, R. R. Cra1g, and F. D. Stul1

\begin{abstract}
AESTRACT
Calculations have been made of the three-dimensional mixing, chemical reaction, and flow field development in a typical ducted rocket configuration. The governing partial differential equations are numerically solved by an iterative finite-difference solution procedure. The physical models include the $k \sim \varepsilon$ turbulence model, one-step reaction, and mixing controlled chemical reaction rate. Radiation is neglected. The mean flow structure, fuel dispersal patterns, and temperature fleld are presented in detail for a base configuration with $0.058 \mathrm{~m}(2 \mathrm{in.})$ dome height, $45^{\circ}$ side arm Inclination, and with gaseous ethylene injected from the dome plate at an eccentric location. In addition, the influences of the geometrical parameters such as dome height, inclination of che side arms, and location of the fuel injector are studied.
\end{abstract}

\section{INTRODUCTION}

A ducted rocket is a ramjet variant with a configuration such as that shown in Figure 1. Gaseous fuel from a gas generator is injected through the dome plate and the air is supplied through two side arms attached to the combustor periphery. The side arms are inclined with the duct axis and are located symmetrically in the azimuthal direction. The mixing of the fuel and air streams occurs in a complex flow fleld formed by the two flow streams. The complex flow recirculation patterns in the dome region and behind the air stream aid in stabilizing the combustion process. A detailed understanding of the aerodynamics and fuel-air mixing processes in such a configuration 18 necessary for improving the combustion efficiency and the 
thrust produced by the ducted rocket. The geometrical variables (such as the angle of the side arms, the length of the combustor, the distance between the dome plate and the side arms, and the location of the fuel injector) may then be optimized for maximum efficiency and thrust.

Numerous flow complexities exist in the ducted rocket configuration. The side entry of the afr stream sets up a complex three-dimensional flow pattern, consisting of a pair of vortices in the cross-sectional plane and a complex recirculation pattern in the dome region. The flow often is highspeed with a choked fuel jet at the dome. The shear layers and the regions of impingement of the alr streams are characterized by intense turbulence and mixing and very little is known of the turbulence-chemistry interactions in such a complex three-dimenstonal flow field. In addition, in a practical ducted rocket, the gas generator effluent has a complex composition which could influence significantly the transient processes such as ignition and flame blowout.

There have been very few studies, experimental or analytical, of the detalled combustion processes inside the ducted rocket. In a continuing investigation, flow visualization studies are being made at the Wright Patterson AFB to characterize the 1sothermal flow flelds. Studies so far [1,2] have Indicated the presence of complex vortex patterns and multiple recirculation regions, espectally in the dome region. Vanka, Stull, and Craig [3] have reproduced some of these flow patterns in an analytical study by numerically solving the partial differential equations governing the steady three-dinensional isothermal fluid flow. Calculations were made for different angles of the side arms and for different dome heights. This preliminary study demonstrated the utility of a computational tool in understanding the ducted rocket combustion processes.

The combustion efficiency for a ducted rocket configurat' ", gaseous athylene injected from the dome was measured in a thrust "tand by Cralg [4] for different fuel air ratios and combustor lengths. The tests were aimed primarily at acquiring the gross features and so did not provide the complete details of the combustion processes and flow fields. Recently, Chen and Tao [5] simplified the ducted rocket geometry to be axisymmetric 
and numerically solved the two-dimenstonal reacting flow equations. Because of the axisymmetric approximation, the study of Chen and Tao [5] does not reveal the vortex structures observed in the water tunnel experiments $[1,2]$. Also, their study neglects the clrcumferential nonuniformities and transport, and therefore inaccurately represents the flow and combustion processes.

The present study has been undertaken to analytically study the characteristics of the three-dimensional reacting flow field in ducted rocket configurations. In this study, equations governing the fullyelliptic three-dimensional reacting flow have been solved numerically by an iterative finite-difference algorithm. For simplicity, the combustion process is assumed to occur in a one-step fast chemical reaction, represented as

$1 \mathrm{~kg}$ fuel $+1 \mathrm{~kg}$ oxidant $=(1+1) \mathrm{kg}$ products,

where $i$ is the stolchiometric oxidant-to-fuel ratio. The chemical reaction is taken to be mixing-limited and fuel and oxidant are assumed to react instantaneously. The effects of turbulence are represented through a turbulence model in which a scalar eddy viscosity is calculated from two local turbulence variables. In the current work, the $(k \sim \varepsilon)$ turbulence model [6] has been used and partial differential iquations are solved for the transport of the kinetic energy of turbulence, $k$, and 1 ts dissipation rate, $E$. Radiation is neglected. These simplifications have been made primarily to capture the Important features of the flow without extensive computation. In the present work, calculations also have been made for different geometrical parameters to study their influence on flow fleld development and mixing efficiency.

The following sections describe the details of the current calculations. The governing equations are given in Section 2 and the solution algorlthm is described briefly in section 3. The results of the calculations for a base case of $0.058 \mathrm{~m}$ ( $2 \mathrm{ln}$.$) dome position and 45^{\circ}$ side arm angle, corresponding to the experiments of Craig [4], are given in Section 4. In Section 5, the effects of varying the side arm angle, dome helght, and location of the fuel injector are investigated. 


\section{GOVERNING BQUATIOAS}

The ducted rocket geometry is conveniently described in the cylindrical polar coordinate system. Th" alr flow from the side arms is prescribed as a boundary condition to the flow domain; consequently, the flow in the sice arms is not analyzed. Because flow recirculation is in all three space directions, the Fully elliptic three-dimensional steady-state Navier-Stokes equations, given below, are solved.

\section{Uass continuity}

$$
\frac{\partial}{\partial \mathbf{x}}(\rho \mathbf{u})+\frac{\partial}{\mathbf{r} \partial \mathbf{r}}(\mathbf{r} \rho \mathbf{v})+\frac{\partial}{\mathbf{r} \partial \theta}(\rho \mathbf{w})=0
$$

\section{$\underline{x-\text { prentun }}$}

$$
\begin{aligned}
\frac{\partial}{\partial x}(\rho u u) & +\frac{\partial}{r \partial r}(r \rho v u)+\frac{\partial}{r \partial \theta}(\rho w u)=-\frac{\partial p}{\partial x}+\frac{\partial}{\partial x}\left(\mu_{\text {eff }} \frac{\partial u}{\partial x}\right) \\
& +\frac{\partial}{\partial r}\left(r \mu_{\text {eff }} \frac{\partial u}{\partial r}\right)+\frac{\partial}{r \partial \theta}\left(\mu_{\text {eff }} \frac{\partial u}{r \partial \theta}\right)+s_{u}
\end{aligned}
$$

$\underline{\text { r-momentum }}$

$$
\begin{aligned}
\frac{\partial}{\partial x}(\rho u v) & +\frac{\partial}{r \partial r}(r \rho v v)+\frac{\partial}{r \partial \theta}(\rho w v)=-\frac{\partial p}{\partial r}+\frac{\partial}{\partial x}\left(\mu_{\text {eff }} \frac{\partial v}{\partial x}\right) \\
& +\frac{\partial}{r \partial r}\left(r \mu_{\text {eff }} \frac{\partial v}{\partial r}\right)+\frac{\partial}{r \partial \theta}\left(\mu_{\text {eff }} \frac{\partial v}{r \partial \theta}\right)+s_{v}
\end{aligned}
$$

\section{$\theta$-momentun}

$$
\begin{aligned}
\frac{\partial}{\partial x}(\rho u w) & +\frac{\partial}{r \partial r}(r \rho v w)+\frac{\partial}{r \partial \theta}(\rho w w)=-\frac{\partial p}{r \partial \theta}+\frac{\partial}{\partial x}\left(\mu_{\text {eff }} \frac{\partial w}{\partial x}\right) \\
& +\frac{\partial}{r \partial \theta}\left(r \mu_{\text {eff }} \frac{\partial w}{\partial r}\right)+\frac{\partial}{r \partial \theta}\left(\mu_{\text {eff }} \frac{\partial w}{r \partial \theta}\right)+s_{w}
\end{aligned}
$$

The turbulent diffusional fluxes are calculated from a two-equation $(k \sim E)$ turbulence model. The additional equations solved are: 
5

Kinetic energy of turbulence (k)

$$
\begin{aligned}
\frac{\partial}{\partial \mathbf{x}}(\rho \mathrm{uk}) & +\frac{\partial}{\mathbf{r} \partial \mathbf{r}}(\rho \mathrm{rvk})+\frac{\partial}{\mathrm{r} \partial \theta}(\rho w k)=\frac{\partial}{\partial \mathbf{x}}\left(\Gamma_{k} \frac{\partial k}{\partial \mathbf{x}}\right)+\frac{\partial}{\partial \mathbf{r}}\left(\mathbf{r} \Gamma_{k} \frac{\partial k}{\partial \mathbf{r}}\right) \\
& +\frac{\partial}{\mathbf{r} \partial \theta}\left(\Gamma_{k} \frac{\partial k}{r \partial \theta}\right)+P-\rho \varepsilon
\end{aligned}
$$

Rate of dissipation of $k$

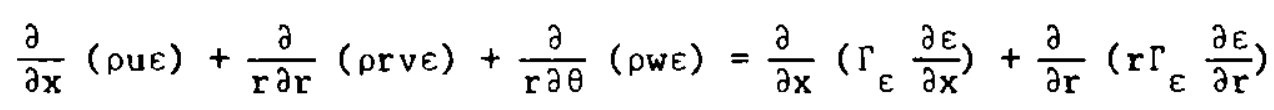

$$
\begin{aligned}
& +\frac{\partial}{\mathrm{r} \partial \theta}\left(\Gamma_{\varepsilon} \frac{\partial \varepsilon}{\mathrm{r} \partial \theta}\right)+\mathrm{C}_{1} \mathrm{P} \varepsilon / \mathrm{k}-\mathrm{C}_{2} \rho \varepsilon^{2} / \mathrm{k}
\end{aligned}
$$

The turbulent viscosity $\mu_{t}$ is calculated from the relation

$$
\mu_{t}=C_{\mu} \rho \hat{k}^{2} / \varepsilon
$$

The chemical reaction is assumed to be fast and to occur in one step. The combustion process therefore is limited by the mixing of the fuel and air streams. The mixing of the two streams is calculated by solving an equation for a conserved scalar, defined as

$$
\mathrm{f}=\frac{\Phi-\Phi_{A}}{\Phi_{F}-\Phi_{A}} \text {, }
$$

where $\Phi=\mathrm{m}_{\mathrm{fu}}-\mathrm{m}_{\mathrm{ox}} / \mathbf{i}$ (also a conserved scalar) and the subscripts $\mathrm{F}$ and $A$ denote fuel and air streams, respectively. The statistical nature of the mixing is represented through an a prior i probability density function. Transport equations for the mean (f) and variance ( $g$ ) of the conserved scalar are solved $[7,8]$. These are:

$$
\begin{aligned}
\frac{\partial}{\partial x}(\rho \mathrm{uf})+\frac{\partial}{r \partial r}(r \rho v f) & +\frac{\partial}{r \partial \theta}(\rho w f)=\frac{\partial}{\partial x}\left(\Gamma_{f} \frac{\partial f}{\partial x}\right)+\frac{\partial}{\partial r}\left(r \Gamma_{f} \frac{\partial f}{\partial r}\right) \\
& +\frac{\partial}{r \partial \theta}\left(\Gamma_{f} \frac{\partial f}{r \partial \theta}\right) \\
\frac{\partial}{\partial x}(\rho \text { fug })+\frac{\partial}{r \partial r}(r \rho v g) & +\frac{\partial}{r \partial \theta}(\rho w g)=\frac{\partial}{\partial x}\left(\Gamma_{g} \frac{\partial g}{\partial x}\right)+\frac{\partial}{\partial r}\left(r \Gamma_{g} \frac{\partial g}{\partial r}\right) \\
& +\frac{\partial}{r \partial \theta}\left(\Gamma_{g} \frac{\partial g}{r \partial \theta}\right)+C_{g l} P_{g}-C_{g 2} \frac{\rho \varepsilon g}{k}
\end{aligned}
$$


The various constants in the turbulence models are given the following values [6]:

$$
\begin{aligned}
& \mathrm{C}_{\mu}=0.09, \mathrm{C}_{1}=1.47, \mathrm{C}_{2}=1.92, \mathrm{c}_{\mathrm{g} 1}=2.8, \mathrm{C}_{\mathrm{g} 2}=2.0, \\
& \sigma_{\varepsilon}=1.3, \sigma_{\mathrm{k}}=1.0, \sigma_{\mathrm{f}}=0.6, \sigma_{\mathrm{g}}=0.6
\end{aligned}
$$

The expresstons for the additional source terms and the exchange coefficients are given in Table 1.

The fluld properties, such as density, spectfic heat, etc., are evaluated from the temperature fleld, which is calculated from the distribution of the mixture fraction and its variance. A battlement shaped probability density function is assumed aud the maximum and minimum values of $f$ at any point, $f_{+}$and $f_{-}$, are represented by

$$
\begin{aligned}
& f_{+}=f+g^{l / 2} \\
& f_{-}=f-g^{1 / 2}
\end{aligned}
$$

except where the value of $f_{+}$exceeds unity and where the value of $f_{-}$is less than zero. In regions where $f_{+}$exceeds unity and $f_{-}$is less than zero, a factor, $B$ is defined by

$$
f=B f_{+}+(1-\beta) f_{-}
$$

where $B$ represents the proportion of time spent in the $f_{+}$state. Values of temperature and the mass fractions of fuel and oxygen are ralculated corresponding to $f_{+}$and $f_{-}$and the mean quantities are ohtalned from the corresponding $T_{+}, T_{-}, m_{f u_{+}}, m_{f u_{-}}, m_{o x_{+}}$, and $m_{o x_{-}}$together with $B$. The density of the mixture of air, the combusting gas, and the combustion products is represented by the equation of a perfect gas:

$$
\rho=\frac{M}{R} \mathrm{~T}
$$


where $M$ is the mixture molecular weight and is calculated from the relation

$$
\frac{1}{M}=\frac{m_{f u}}{M_{f u}}+\frac{m_{o x}}{M_{o x}}+\frac{m_{p r}}{M_{p r}} .
$$

The mixture specific heat is calculated from a linear combination of the component specific heats. Thus,

and

$$
c_{p}=\sum_{i} m_{i} c_{p 1}
$$

$$
c_{p i}=a_{i}+b_{i} T+c_{1} T^{2}
$$

The stagnation enthalpy f.s defined as

$$
h=m_{f u} H_{f u}+C_{p} T+\frac{1}{2}\left(u^{2}+v^{2}+w^{2}\right) .
$$

where $\mathrm{H}_{\mathrm{fu}} \mathrm{is}$ the heat of reaction. The values currently assigned to the constants in specific heat relations, molecular weights, etc., are given in Table 2 and correspond to those of gaseous ethylene $\left(\mathrm{C}_{2} \mathrm{H}_{4}\right)$.

\section{SOLUTION ALGORITHI}

The set of partial differential equations in Section 2 is solved by an tterative fintte-difference algorithm, SIMPLE, developed by Patankar and Spalding [9]. The partial differential equations are integrated over small discrete reglons called control volumes, and are converted to a set of nonlinear algebraic equations. The nonlinear algebralc equations are derived for the primitive variables $u, v, w$, and $p$. A staggered mesh system is employed in locating the flow variables on the finite-difference grid, and an exponential inter node variation of the variable is assumed for the purpose of evaluating the fluxes from the faces of the control volumes.

The nonlinear algebraic equations are solved in a decoupled manner. The momentum equations are first solved using an estimated pressure field. The estimated pressure fleld is then updated by solving a pressurecorrection equation, derived by combining the momentum and continuity equations. To prevent numerical instability, the successive changes of the 
flow variables are underrelaxed with their old values. The new value of a general varlable, $\phi$, 1s taken to be

$$
\phi=\alpha \phi^{n}+(1-\alpha) \phi^{0},
$$

where $\phi^{\mathrm{n}}$ is the value computed with no underrelaxation and $\phi^{\circ}$ is the old iterate value. $\alpha$ is the underrelaxation factor which has a value between 0 and 1. The general structure of the final finite-difference equation is

$$
A_{P} \phi_{P}=\sum_{n} A_{n} \phi_{n}+s^{u}+S^{p} \phi_{P}
$$

where $A_{P}$ and $A_{n}$ are the finite-difference coefficients for point $P$ and its six neighbors. $\mathrm{S}^{\mathrm{u}}$ and $\mathrm{S}^{\mathrm{p}}$ are the integrated source terms, $\mathrm{S}^{\mathrm{p}}$ being the linearized part. Equation 21 is solved by repeated alternate line sweeps in the three coordfnate directions. At each line, a line Gaussian elimination algorithm is used.

Complete detalls of the SIMPLE algorithm can be obtained from several earlier refeiences, notably from Reference 9. The momentum and continuity equations also can be solved in a fully coupled manner, resulting in a block inplicit method. Such an algorithm has been developed by Vanka [10] for two-dimensional flows and has been found to accelerate the convergence significantly. The block inplicit algorithm has not been extended yet to three-dimensional flows.

\section{CALCULATED FLON AND SCALAR PIELDS}

\subsection{Conputational Aspects}

Because of the complexity of the flow field, the rate of convergence of the calculations has been slow. For this reason, the present calculations were made only with a coarse finite-difference mesh consisting of a modest number $(11 \times 11 \times 24)$ of grid nodes. Because of the symmetric flow, the equations were solved for only half of the cross section. The CPU time required for these calculations was 25 minutes on an IBM 3033 computer. 
Typically 450 iterations were necessary to make the residuals decrease to the $10^{-4}$ level. Because of the large computational times, finer finitedifference meshes could not be used. Nevertheless, the accuracy of the present calculations is suffictent to understand the flow processes in detall, and to draw conclusions about the trends of the influences of the various geometrical parameters.

The conditions for the base configuration, given in Table 3, correspond to the experiments of Craig [4]. Pertuibations have been made to the base configuration to Investigate the influence of the geometrical parameters. The results of the calculations are described in subsequent sections of this chapter.

\subsection{Flow Fleld}

The azimuthal location of the side arms and their inclination with the duct axis create a complex vortex pattern. In the cross-stream, the flow consists of two symmetrical pairs of vortices. Figure 2 shows the development of these cross-sectional vortex structures at several axial distances of the base flow configuration. The vortex structures are more clearly formed downstream of the air entry, although some form of vortex structure is also observed in the dome region. The cross-stream vortex pattern observed in the reacting flow situation is similar to the isothermal flow pattern earller calculated by Vanka et al. [3].

The flow structure in planes of constant azimuthal angle $(\theta)$ is shown in Figure 3. The axlal length is scaled down by a factor of four. The flow field in these planes consists basically of two regions. In the dome region, $t_{. e .,}$ between the afr inlets and the dome plate, the flow consists of low velocity rectrculating eddies. These eddies are formed through part of the atr flow bifurcating into the dome region and interacting with the fuel stream. The flow in this region is truly three-dimenstonal. In the region downstreas of the atr inlet, the flow ts helical, being a superposition of a vortex pattern on an almost unidirectional flow. This flow structure of the azimuthal planes also is similar to the 1sothermal flow fleld in Vanka et al. [3], but some differences exist. In the 
isothermal calculations, regtons of flow recirculation were observed also downstream of the air inlet. These regions are absent in the reacting case. The probable cause for this is the expansion of the gases due to combustion, and the resulting higher flow velocities. It should be noted that although the fiow in the region downstream of the alr Inlets is unidirectional, there can be significant flow ellipticities because of the nonuniformities in the pressure field.

\subsection{Tenperature and Puel Praction Contours}

Figure 4 shows the contours of temperature at selected cross-sectional planes. Because of the diffusion flame assumption, the temperature patterns are linked closely to the fuel-air mixing patterns. The location where the fuel-air ratio is stolchiometric can be interpreted as being on the flame front. For the temperature contours, this corresponds to regions of steep temperature gradients.

Figure 4(a) corresponds to a location in the dome region. Here, the fuel jet is expanding and tuel-air mixing occurs at the boundary of the fuel jet. Further downstream, in Figure 4(b), the hot products are transported by the cross-sectional vortex flow field, and a more uniform temperature field is generated. The higher temperatures are at the left side of the circumference and in the cross-sectional eddy. The location $x=0.0889 \mathrm{~m}$ [Figure 4(c)] corresponds to the downstream edge of the air inlet. The air entry is froin tie right quadrant of the figure and is reflected in lower $\left(\begin{array}{lll}\sim & \mathrm{K}\end{array}\right)$ tenperatures. The penetration of the alr stream into the combusting mixture can be seen in Figures $4(d)$ and $4(e)$, characterized by lower temperatures. Figure $4(\mathrm{f})$ shows the temperature pattern that $1 \mathrm{~s}$ formed after these complex mixing and reaction processes. The largest temperatures occur on the wall opposite to the alr strean closer to the central plane, and near the fuel injection port. This behavior is in agreement with observed surface heating patterns [2].

Figure 5 shows the contours of unburnt fuel fraction at various crosssectional planes. The location of the fuel jet and its dispersal can be 
noticed easily from these plots. The regton in the right quadrant of Figure $5(\mathrm{c})$ corresponds to the atr jet and has a low fuel fraction. The gradual mixing and dimuntition of fuel along the ducted rocket can be seen from Figures 5(a)-5(f). The fuel fractions are higher in the dome region because only a part of the airstream is bifurcated into the dome and is mixed with the fuel. For complete fuel-air mixing and no combustion, the fuel fraction would be 0.0566 . Under conditions of complete combustion, there will be no fuel at the exit of the ducted rocket. In the present situation, some fuel st111 is present at $x=0.2540 \mathrm{~m}$, and even further (not shown here).

Figure 6 shows the cross-sectional average of unburnt fuel fraction plotted against the axial distance (the curves for other dome heights are discussed later). It is seen that the fuel fraction decreases very rapidly in the initial length of the ducted rocket. This region (up to $x \sim 0.3 \mathrm{~m}$ ) is characterized by intense turbulence and mixing. Beyond $x \sim 0.3 \mathrm{~m}$, the fue 1 air mixing and reaction is slow, as reflected in the slow dimunition of the fuel. This slow rate of mixing is attributed to the nearly unidirectional. flow shown earlier in figure 3. The combustion efficiency for this configuration is shown in Figure 7. The combustion efficiency is defined as the ratio of actual enthalpy rise to the ideal value for complete combustion. The combustion efficiency for the present calculations is directly related to the mixing efficiency because of the diffusion flame assumption. The value calculated for this configuration is $84 \%$. This 1 s very close to the value of $84.5 \%$ measured by Craig [4].

A few important conclusions ran be drawn from the above plots. First, the flow fleld in the ducted rocket is very complex, consisting of several recirculation eddies. These recirculation regions are advantageous for producing effictent fuel-air mixing. The flow in the downstream region, however, is almost unidirectional. Second, the temperature distribution in the cross section is severely nonuniform. In the case in which temperaturedependent finite chemical reaction rates are important, these nonuniformities can significantly influence the overall combustion efficiency. Third, abcut $60 \%$ of the effictency is obtatned in one-third of the length and the other $24 \%$ is recovered in the remalning two-thirds of the 
length. It is therefore desirable to investigate alternative configurations that will disturb the strong unidirectional flow. This might require major changes to the base configuration.

\section{INFLUENCE OF GEOAETRICAL PARAMETERS}

Several geometrical and flow parameters could influence the efficiency and the thrust produced by a ducted rocket. These include the dome height, angle of side arms, location of the fuel injector, fuel-air ratio, combustor pressure, and combustor dimensfons. In this section the influence of the first three parameters is considered.

The location and angle of the side arms alter the flow patterns in the dome region signiflcantly. Vanka, Stull, and Craig [3] studied these for the isothermal flow situation. It was observed that shifting the side arms toward the dome plate compresses the eddy in the dome region, and steepening the angle of the arm modifies the recirculation pattern downstream of the air flow and in the dome region. In the present study, calculations have been made to examine the effect of these parameters in a reacting flow case. In addition, calculations are made with the fuel injector located at the center of the dome plate. The air and fuel flow rates and the inlet temperatures are held fixed. The effects of these parameters on the mixing and flow patterns is examined. Also, the average unburnt fraction is plotted agalnst axial distance and is compared for the different cases.

\subsection{Bffect of Done Feight}

The dome helght influences the mixing process through changes to the recirculation flow in the dome region. At $0 \mathrm{~m}$ dome height (1.e., the side arms being flush with the dome plate), the recirculating flow in the dime region is markedly decreased and most of the alr flows directly to the exit nozzle. The mixing $1 \mathrm{~s}$ therefore significantly reduced. The cross stream patterns are similar to the base case; therefore, only the calculated flow patterns in the constant- $\theta$ planes are shown (Figure 8 ). It is seen that the recirculation in the dome region is markedly decreased. The temperature contours at selected locations for this case are shown in Figure 9. 
Figure 10 shows the flow pattern for a larger dome height of $0.116 \mathrm{~m}$ (4 1n.). In this case, the recirculation region of the dome is increased, although it is not clear whether more flow has been bifurcated into the dome. The flow pattern is, however, similar to the base configuration.

The average unburnt fuel fraction and the combustion efficiency for the various dome heights are shown in Figures 6 and 7 . Decreasing the dome height decreases combustion efficlency very little except, for the $0 \mathrm{~m}$ position. The decrease in conbustion efficiency is related directly to the decrease in the recirculation eddy in the dome. Quite surprisingly, it is seen that the combustion efflciency is slightly lower for the dome helght of $0.116 \mathrm{~m}$ (4 in.). Apparently, the Increased length of the dome region does nor increase the bifurcating flow but it decreases overall mixing because of smaller combustor length avallable downstream of the air inlet. It is interesting to observe that the base configuration has the optimum dome height.

\subsection{Effect of Side Arn Angle}

The inclination of the side arm with the duct axis influences the amount of fuel bifurcated into the dome region. The larger this bifurcation flow, the betier the mixing. Steepening the side arm angle increases the flow into the dome, thereby increasing the mixing.

This section examines the quantitative differences in mixing efficiency for three side arm angles- $-60^{\circ}, 45^{\circ}$ (base case), and $30^{\circ}$. Figures 11 and 12 show flow patterns in selected constant- $\theta$ planes for the $60^{\circ}$ and $30^{\circ}$ sidearm inclinations. The dome position and other parameters were held fixed at the base values. The changes in the dome eddy because of changes in sidearm angle are clearly evident in Figures 11 and 12. The cross-stream plots of velocities and concentration and temperature contours are similar to the base calculations but differ somewhat quantitatively.

Figures 13 and 14 summarize the effect of the side arm angle through the plots of unburnt average fuel fraction and combustion efficiency along the combustor length. As expected, there is some improvenent in the mixing 
efficiency when the angle of Inclination is made steeper, to $60^{\circ}$, but flattening the angle to $30^{\circ}$ lowers the efflclency considerably. These changes are purely the result of the modified flow paths.

\subsection{Ef fect of Injector Location}

The location of the Injector alters the interface between the fue 1 and air streams. We have compared here the fuel dispersal patterns for the cases of concentric and eccentric injection of the fuel. In the concentric case, the fuel injector is located at the center of the dome plate, and the other parameters are the same as the base case. Figure 15 shows the flow patterns in the azimuthal planes for the concentric injection case. The qualitative differences between the two flow patterns in the dome region are clearly evident. In the concentric case, the fuel jet is in the regicn of the alr impingement and directly interacts with the air stream. The location of the flame front is therefore also different in this case. The temperature contours for the concentric injection case are shown In Fig. 16.

Figures 17 and 18 show the unburnt fuel fraction and combustion efficiency for the concentric injection case. The overall intxing efficlency has dropped to $70 \%$ from the base value of $84 \%$. Although it was expected that the mixing efficiency might improve when the injector was located in the region of alr implngement, it appears that the fuel jet has created a central adverse pressure gradient, thus decreasing the bifurcating alr flow into the dome region. In the case of the eccentric injection, the fuel jet is not directly in the region where the alr flow bifurcates, so more flow enters the dome, increasing the fuel-air mixing. This is an interesting observation, pointing out the importance of the bifurcating flow in tmproving combustion effictency.

\section{SUMMARY}

In this study, we have analyzed the three dimenstonal flow fleld, fuel dispersal and temperature distributions inside a ducted rocket configuration. The base calculations are made for a $0.1524 \mathrm{~m}$ (6 1n.) diameter ducted rocket wtth $1.81 \mathrm{~kg} / \mathrm{s}(41 \mathrm{bs} / \mathrm{s}))$ of atr and burning ethylene 
at a fuel-air ratio of 0.06. The flow patterns, fuel fraction, and temperature contours are presented. The calculated overall combustion efficiency for the base configuration is in good agreement with the value measured by Craig [4] In a thrust stand. Unfortunately, there are no experimental data to compare the detalled three-dimensional flow and temperature variations. Such data will be helpful in validating the turbulence and combustion models used in this study.

The effect of the dome helght, side arm angle, and injector location are studied. These effects are examined through their impact on the residual fuel fraction and the combustion efficiency. It is observed that the 0.058 in dome height offers the best combustion efficiency compared with $0,0.0254$, and $0.116 \mathrm{~m}$ positions. The mixing and consequent reaction is enhanced by steepening the angle of side arms, and 1 s decreased when the fuel injector is located at the center of the dome plate instead at an eccentric position.

Finally, it should be mentioned that in the present study the effects of several factors such as grid size, inlet turbulence levels, and turbulence model constants have not been investigated thoroughly. Such studies must be undertaken in the future. 


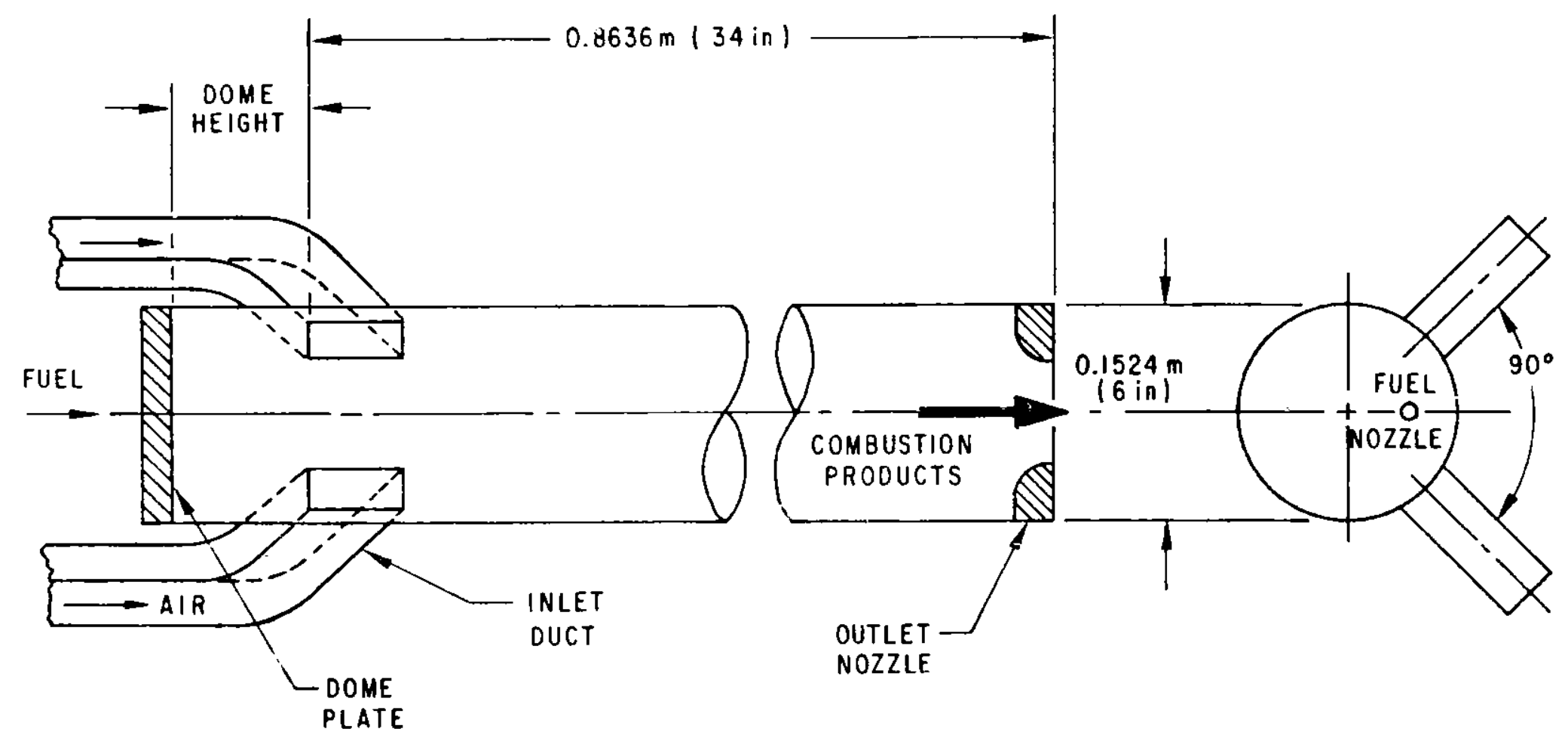

Figure 1. Ducted Rocket Combustor Configuration 
17
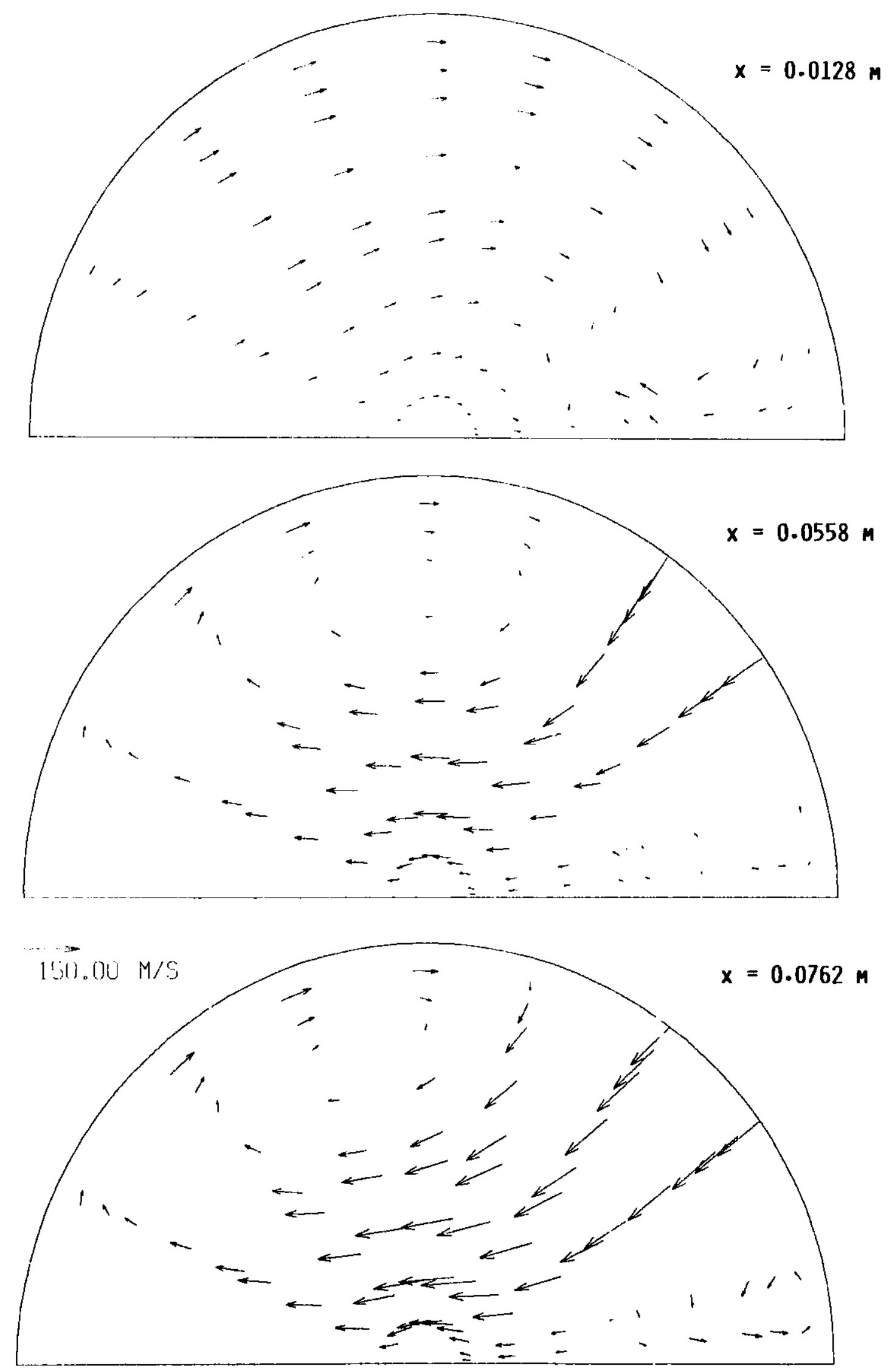

Figure 2. Cross-stream Flow Patterns for Base Configuration 
18
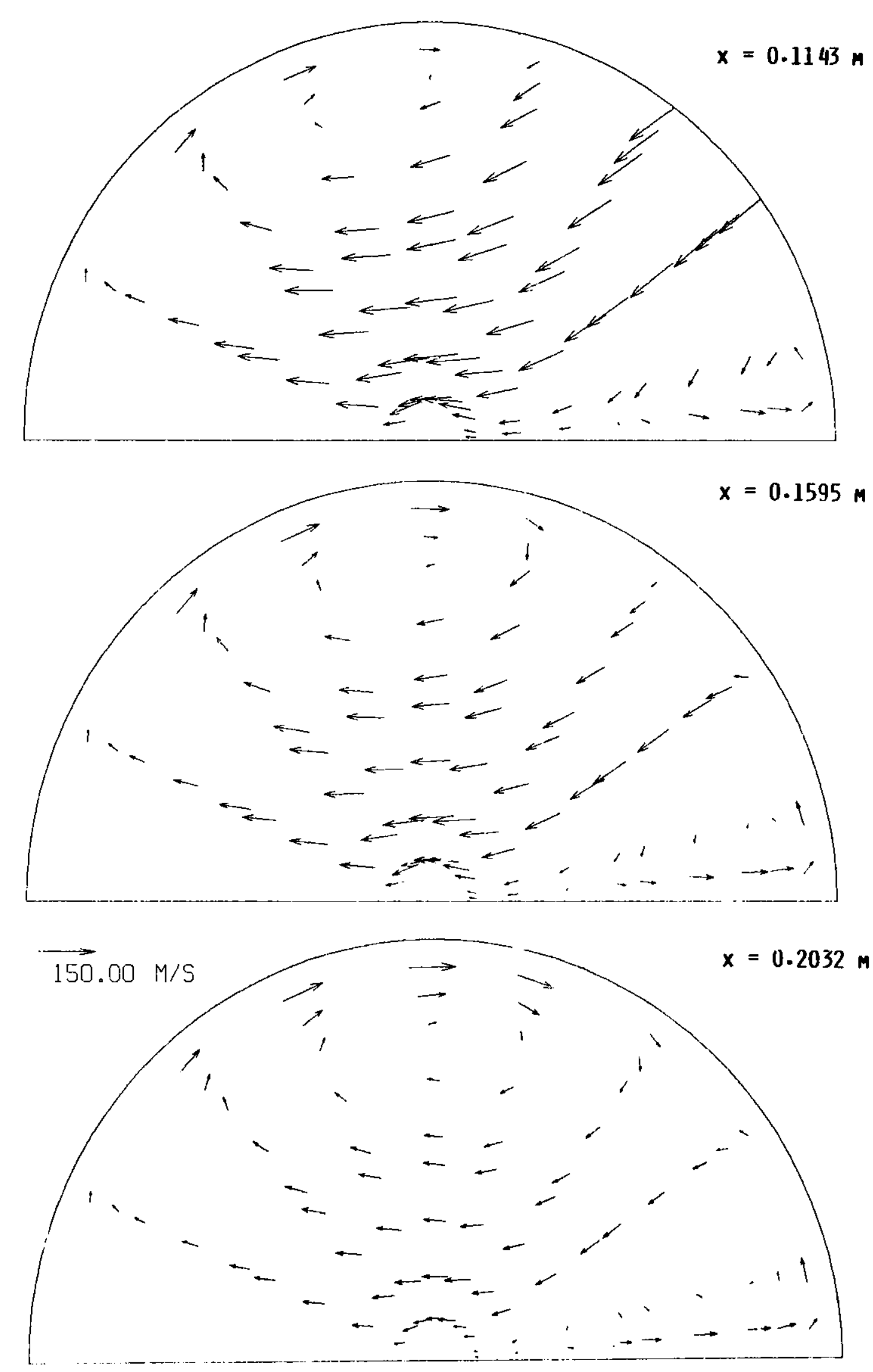

Figure 2. (contd) 

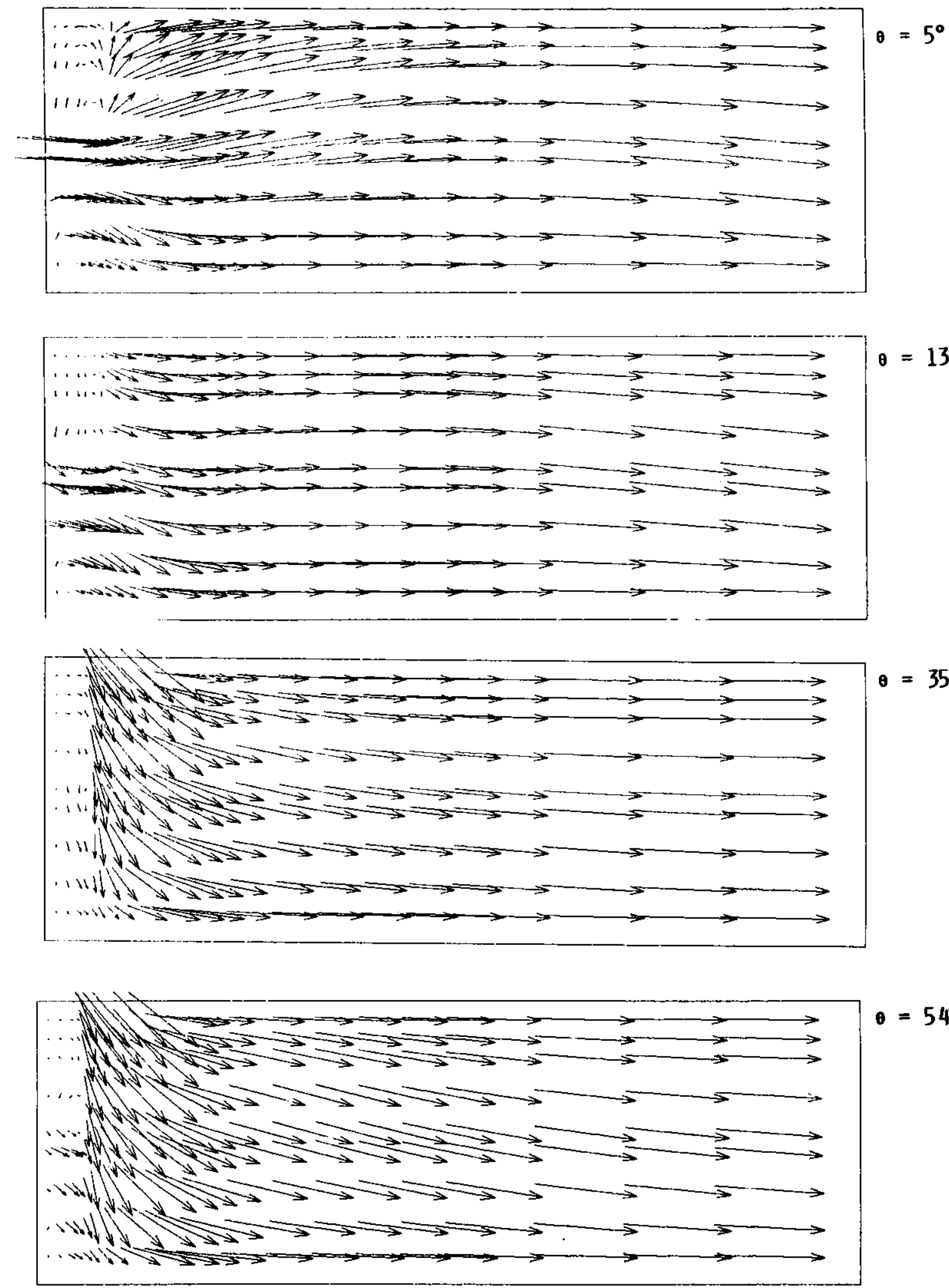

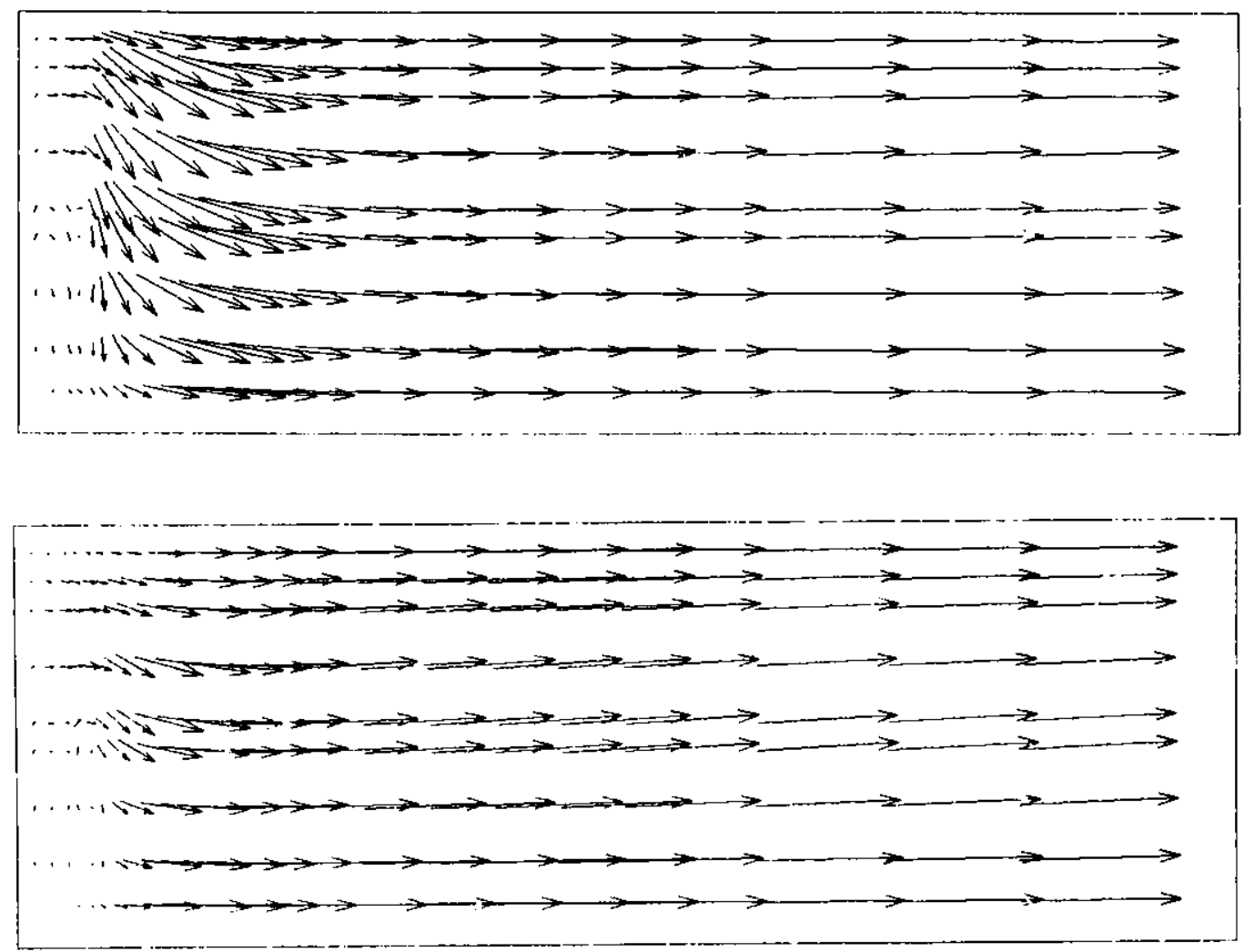

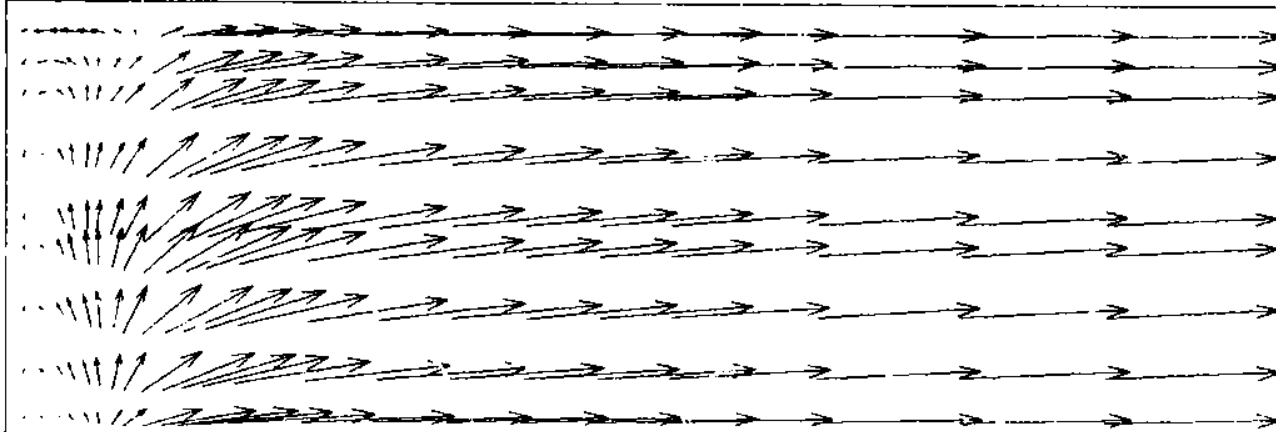

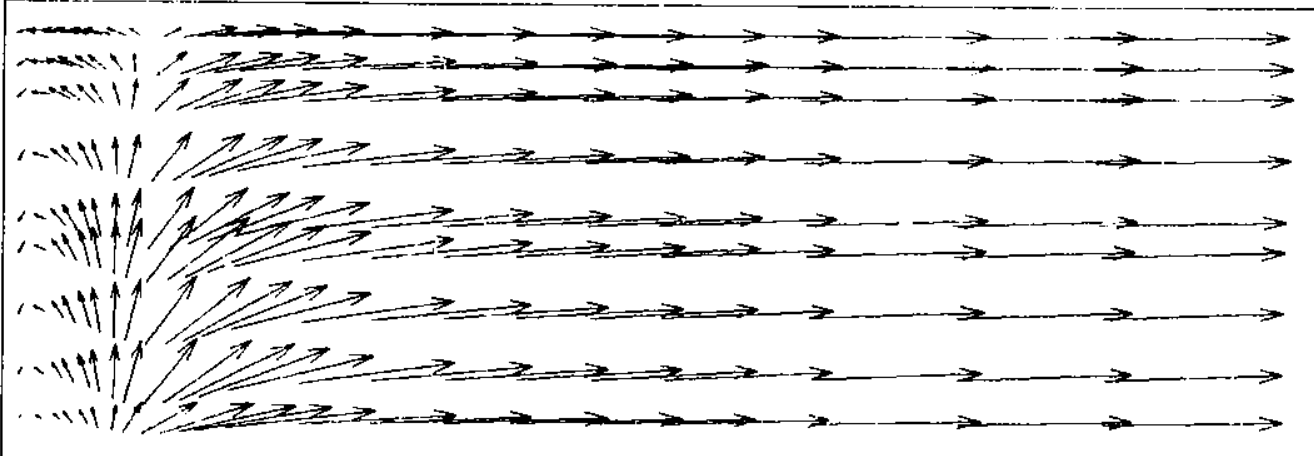



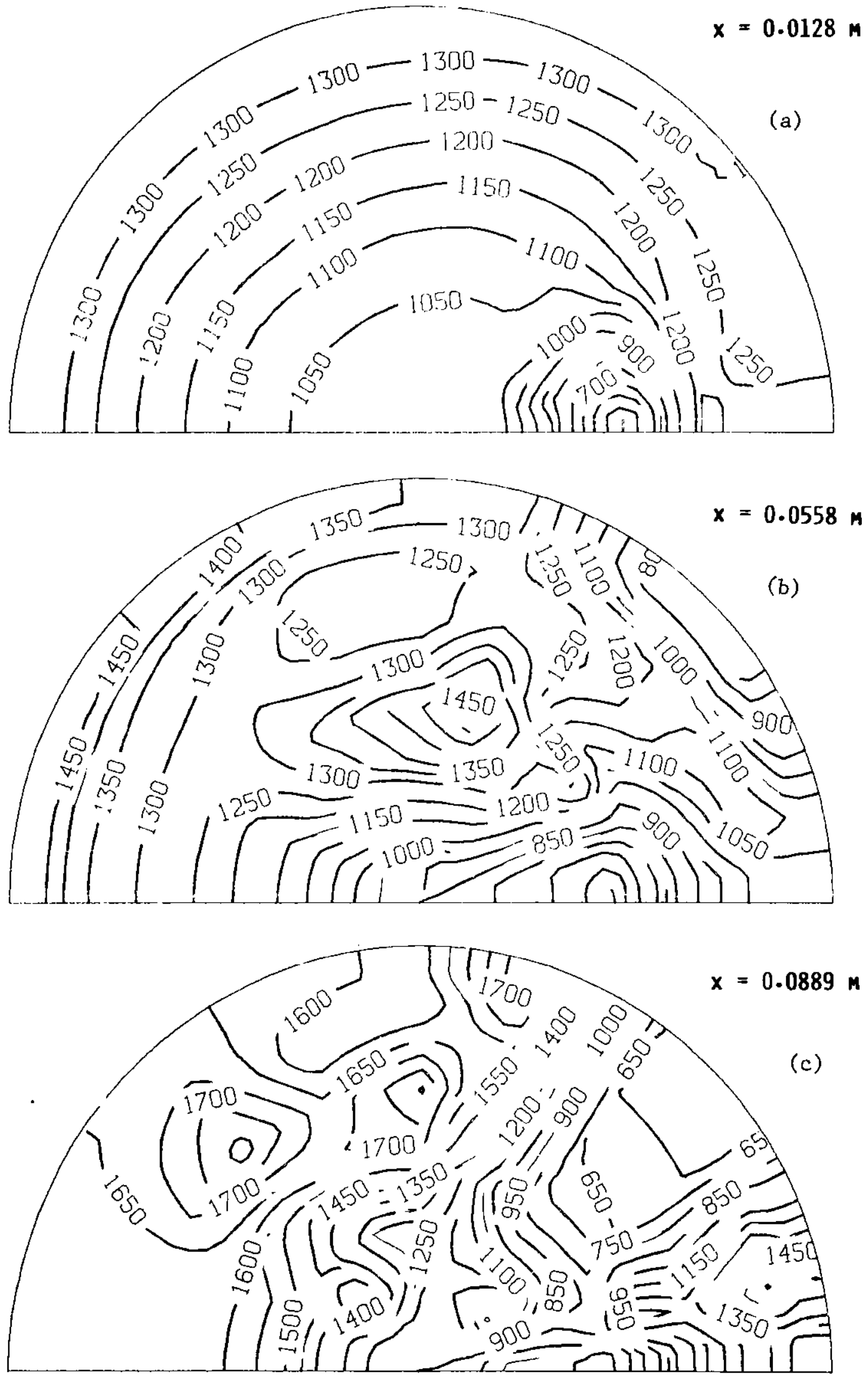

F1gure 4. Cross-stream Temperature Distribution for Base Configuration 

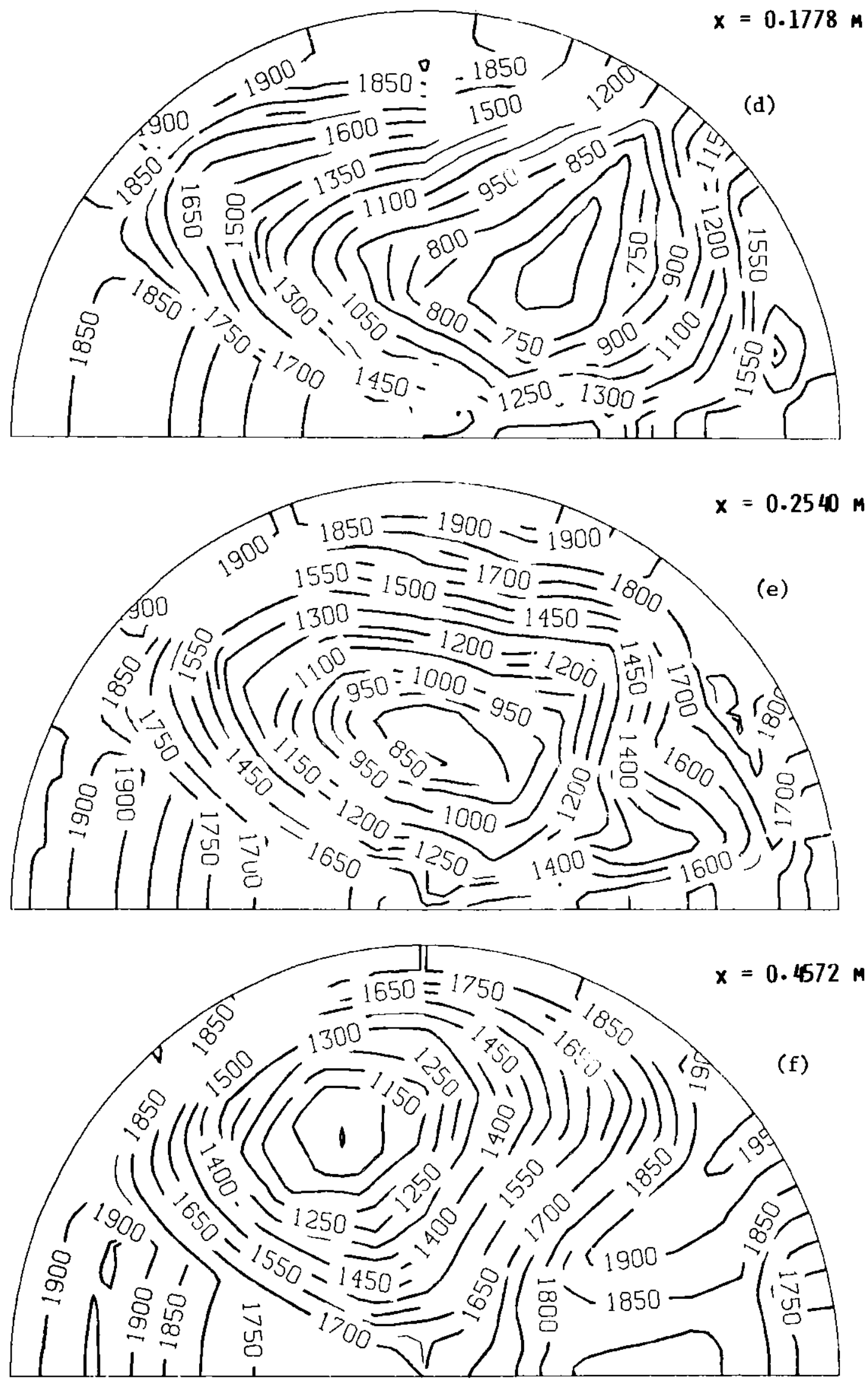

Figure 4. (contd) 

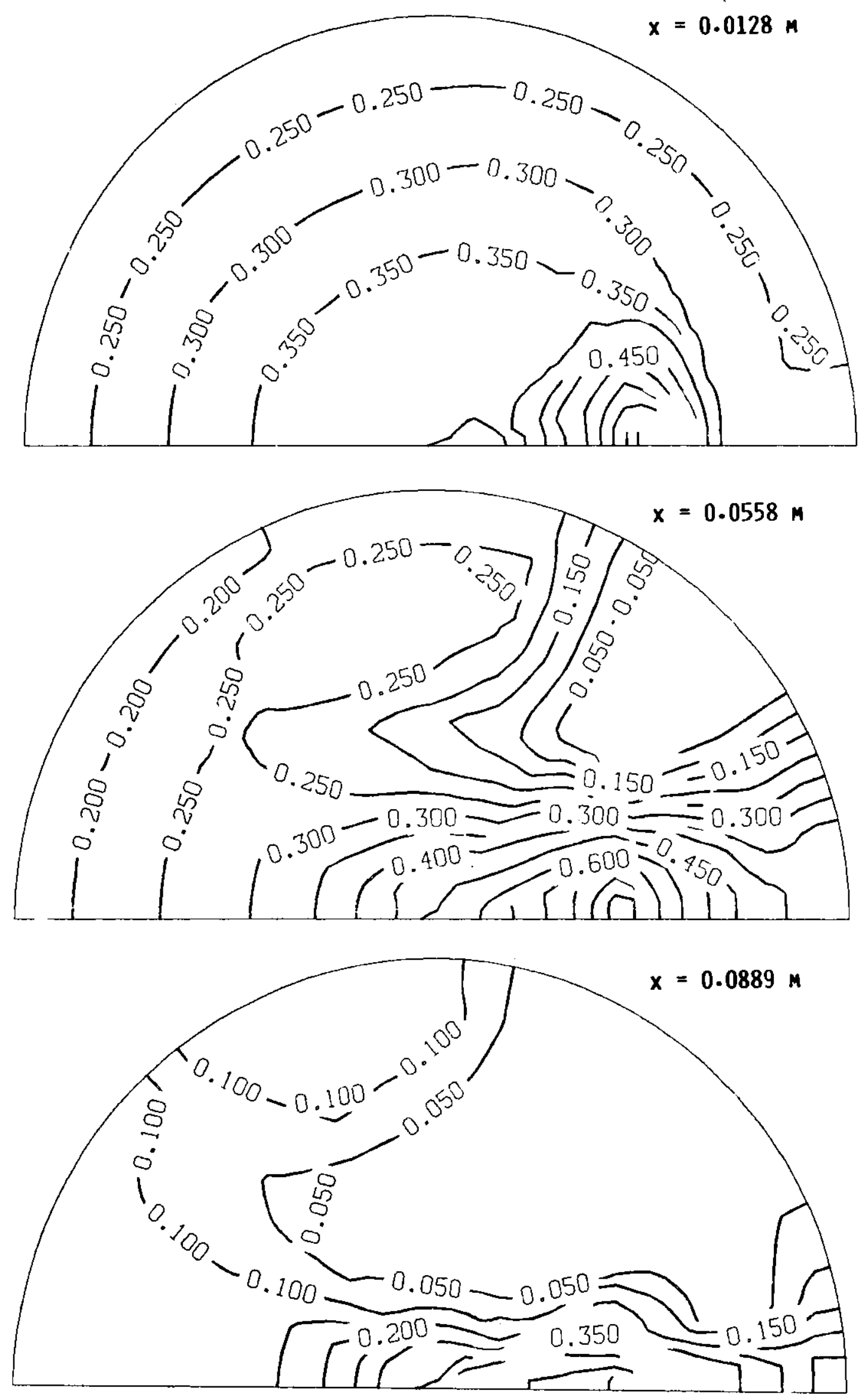

Figure 5. Cross-stream Fuel Fraction Distribution for Base Configuration 

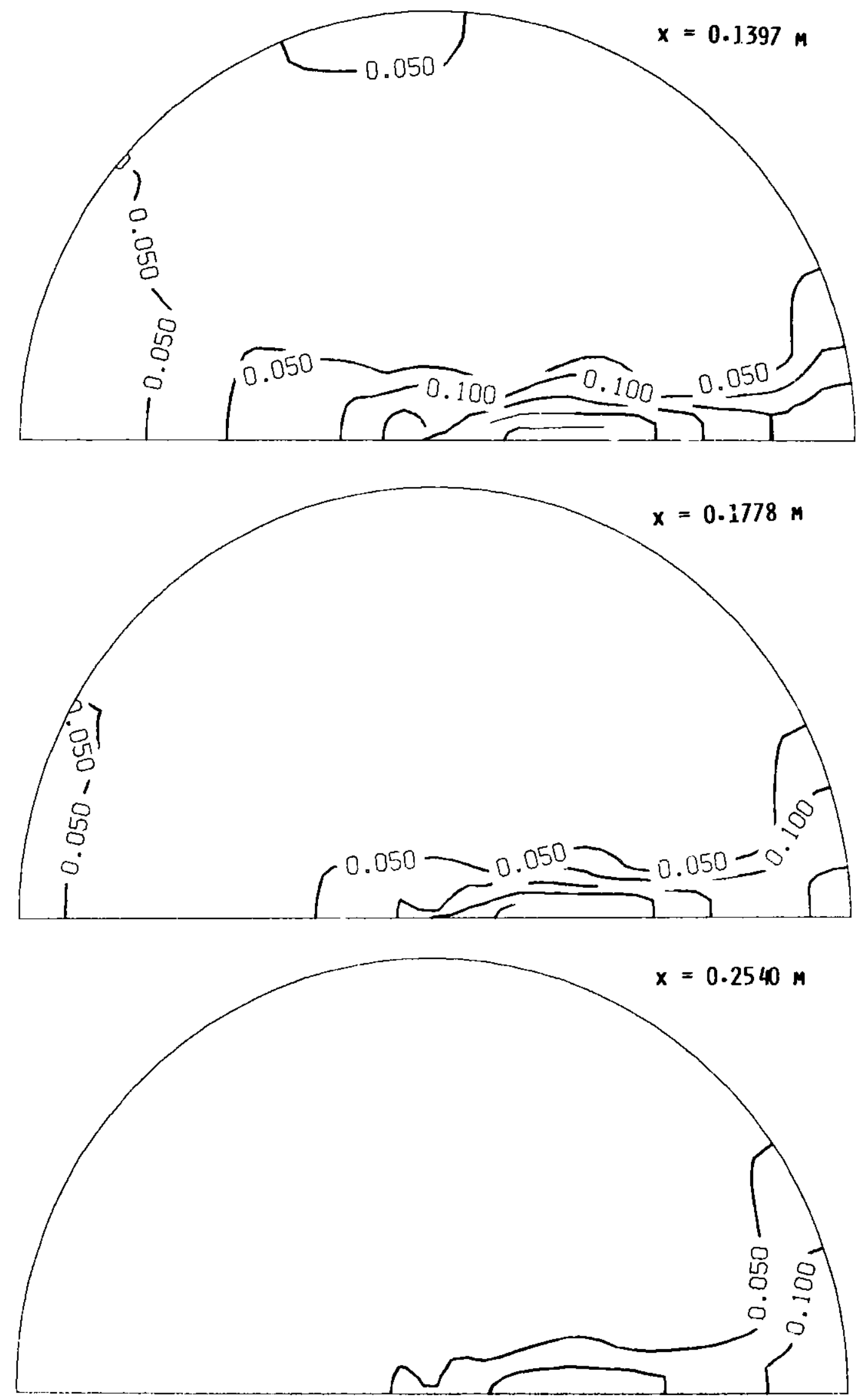

F1gure 5. (contd) 


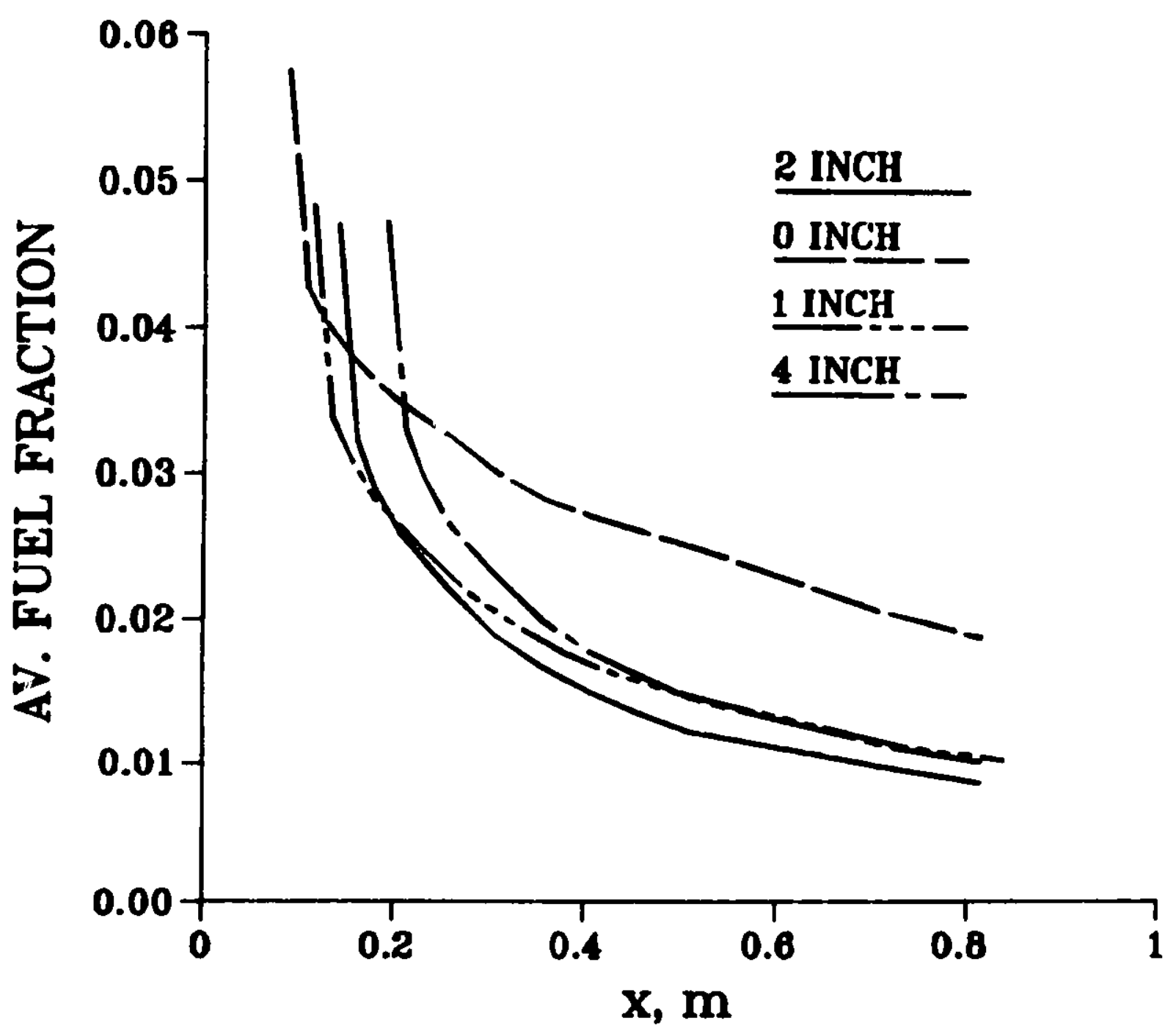

F1gure 6. Axial Variation of Average Fuel Fraction for Base Configuration 


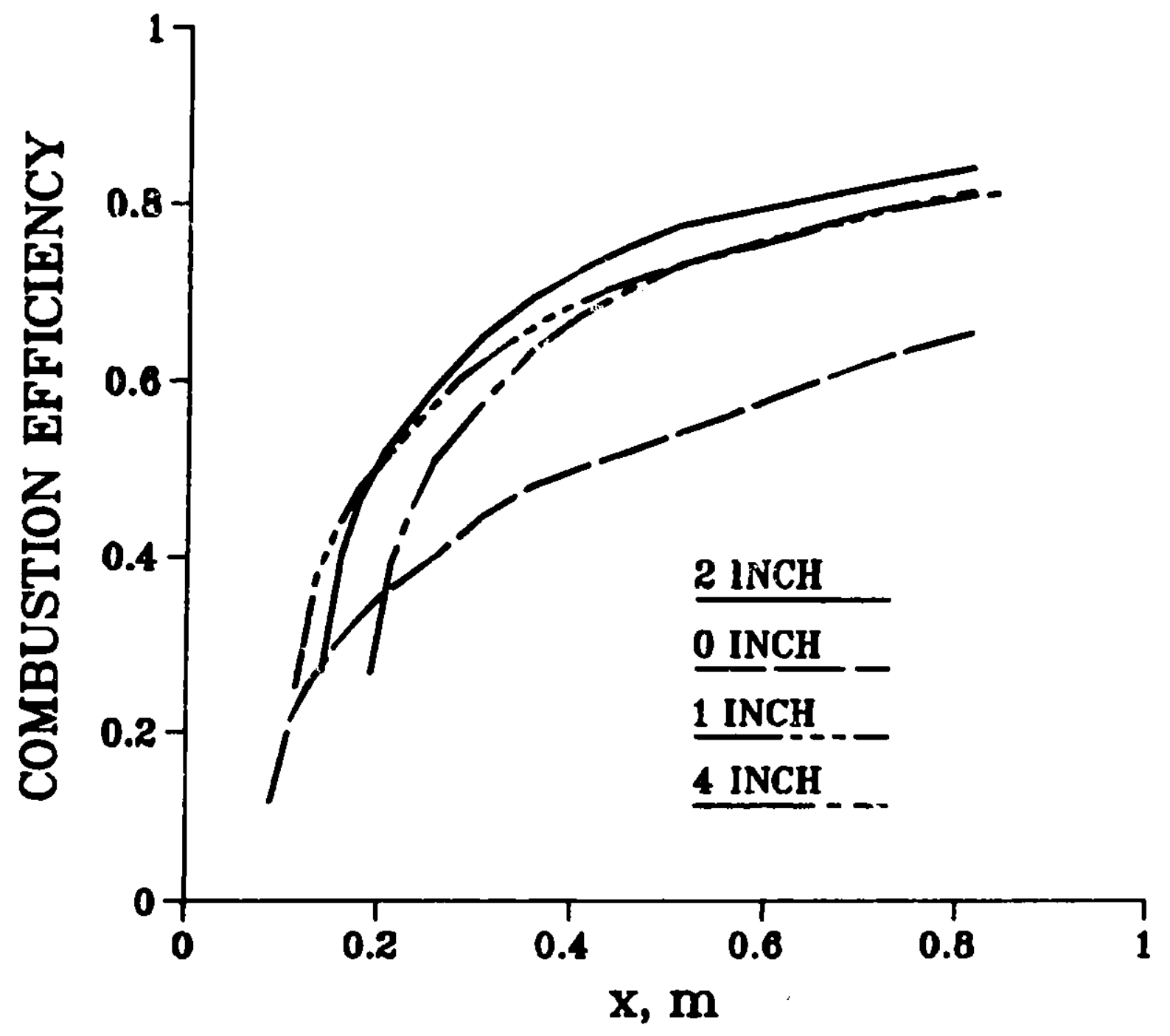

Figure 7. Axial Variation of Combustion Efficiency for Base Configuration 


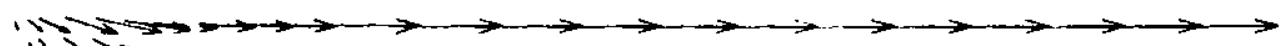

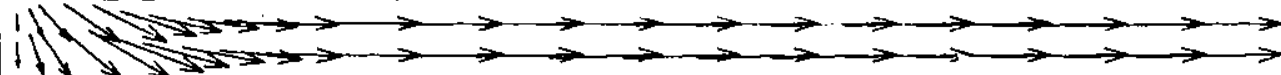

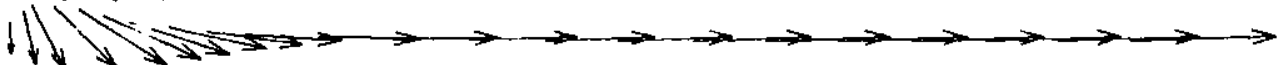

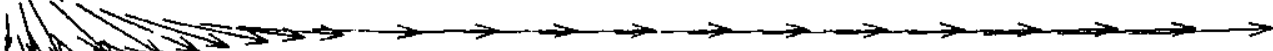

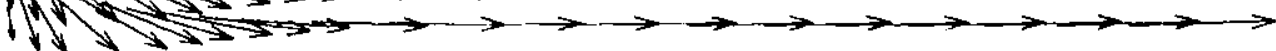

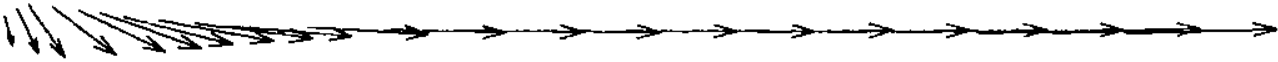

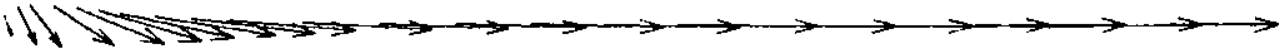

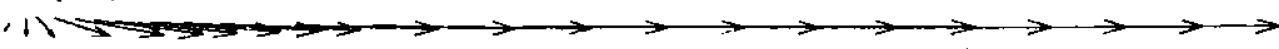

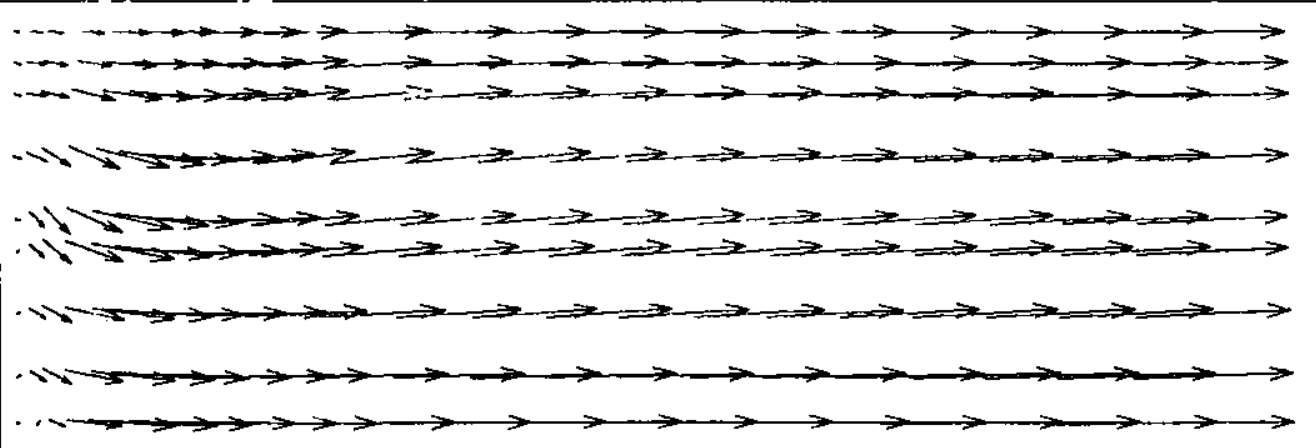

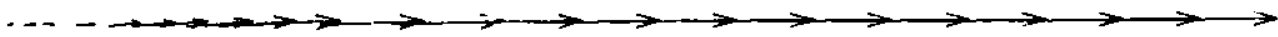

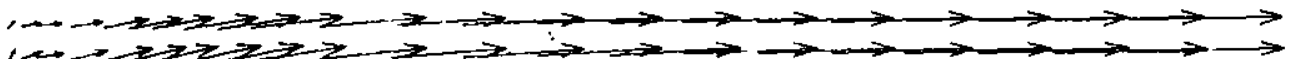

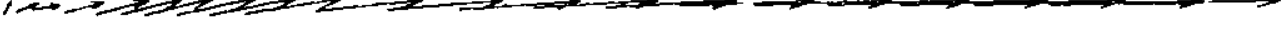

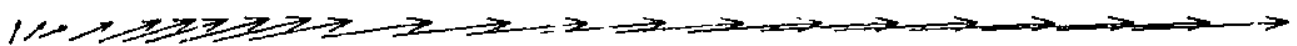

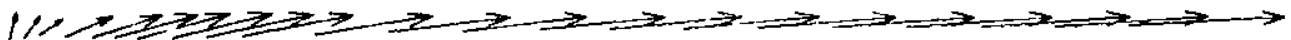

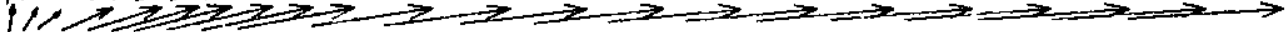

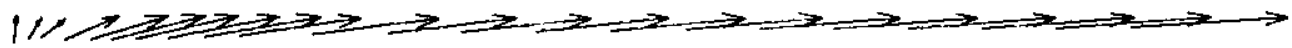

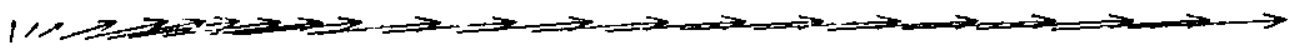

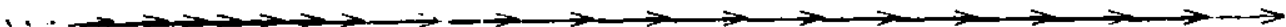

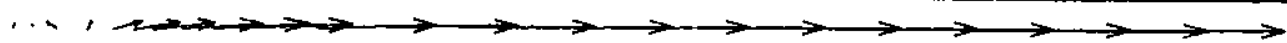

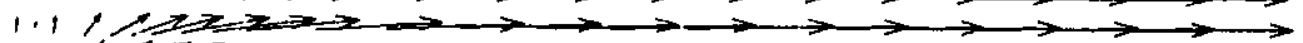

$\theta=155^{\circ}$

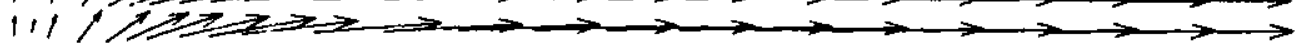

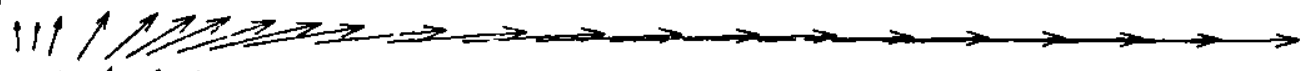

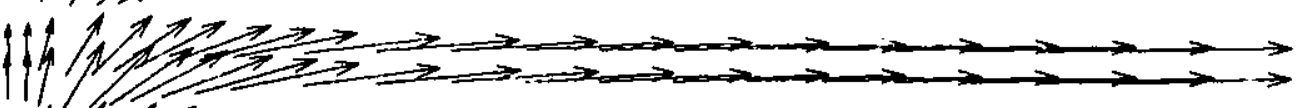

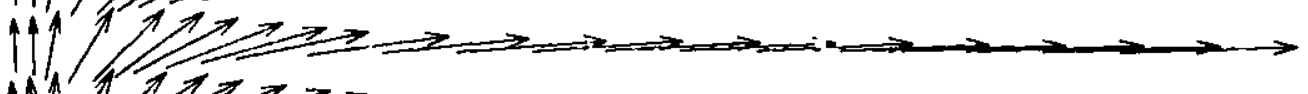

114.

$119>2$

Figure 8. Flow Patterns in Selected Azimuthal Planes for $0.0 \mathrm{~m}$ Dome Height 


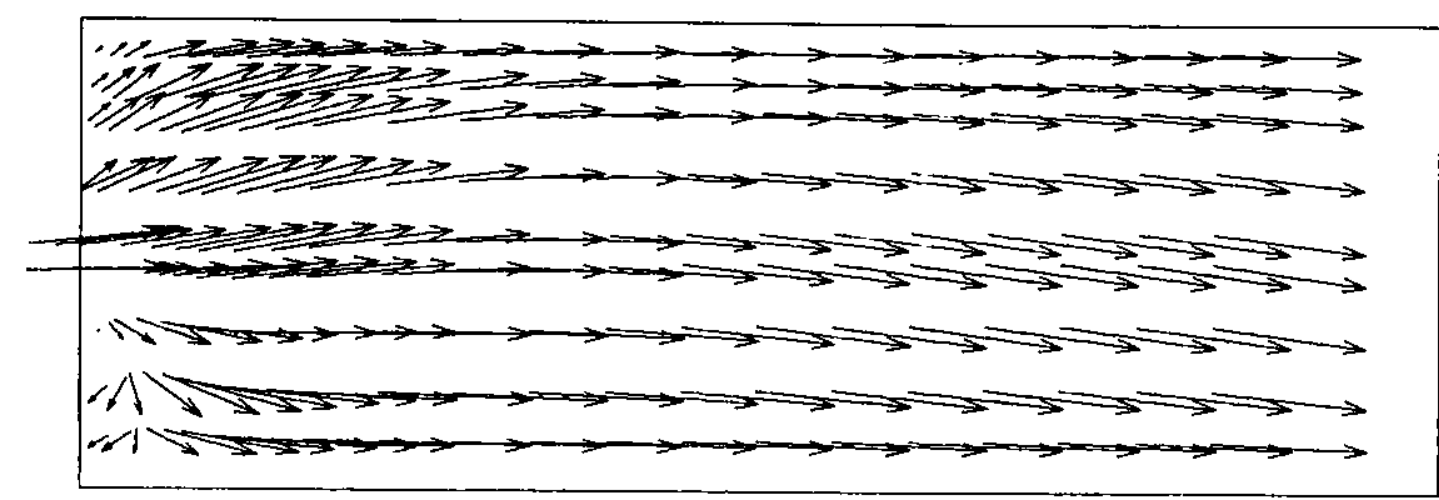

$\theta=5^{\circ}$

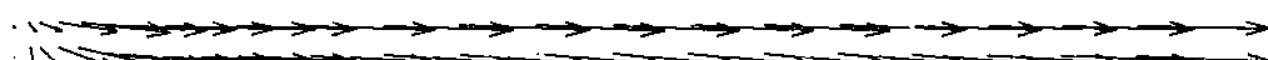
I

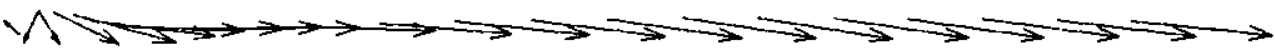

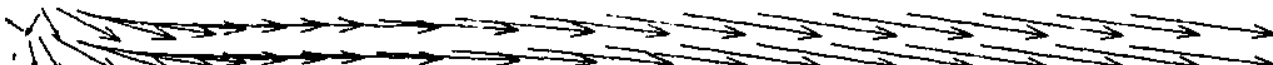

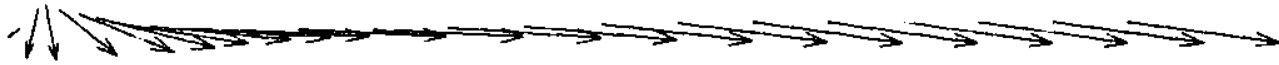

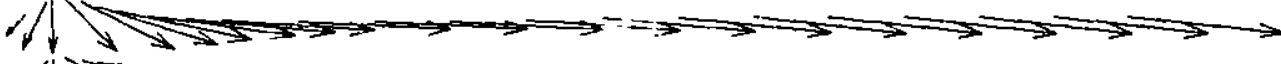
LI $\mathrm{PSOM} \rightarrow \longrightarrow$
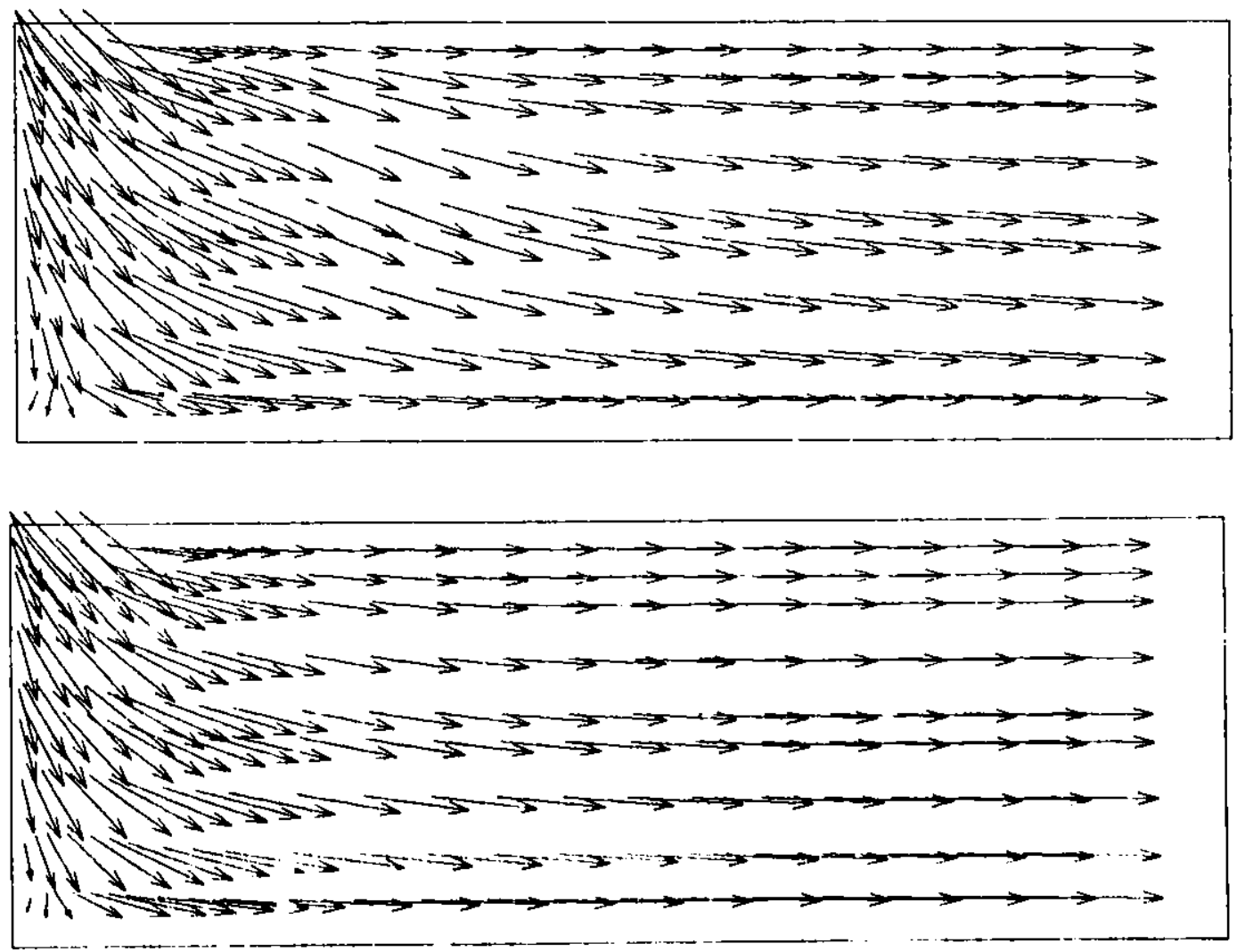

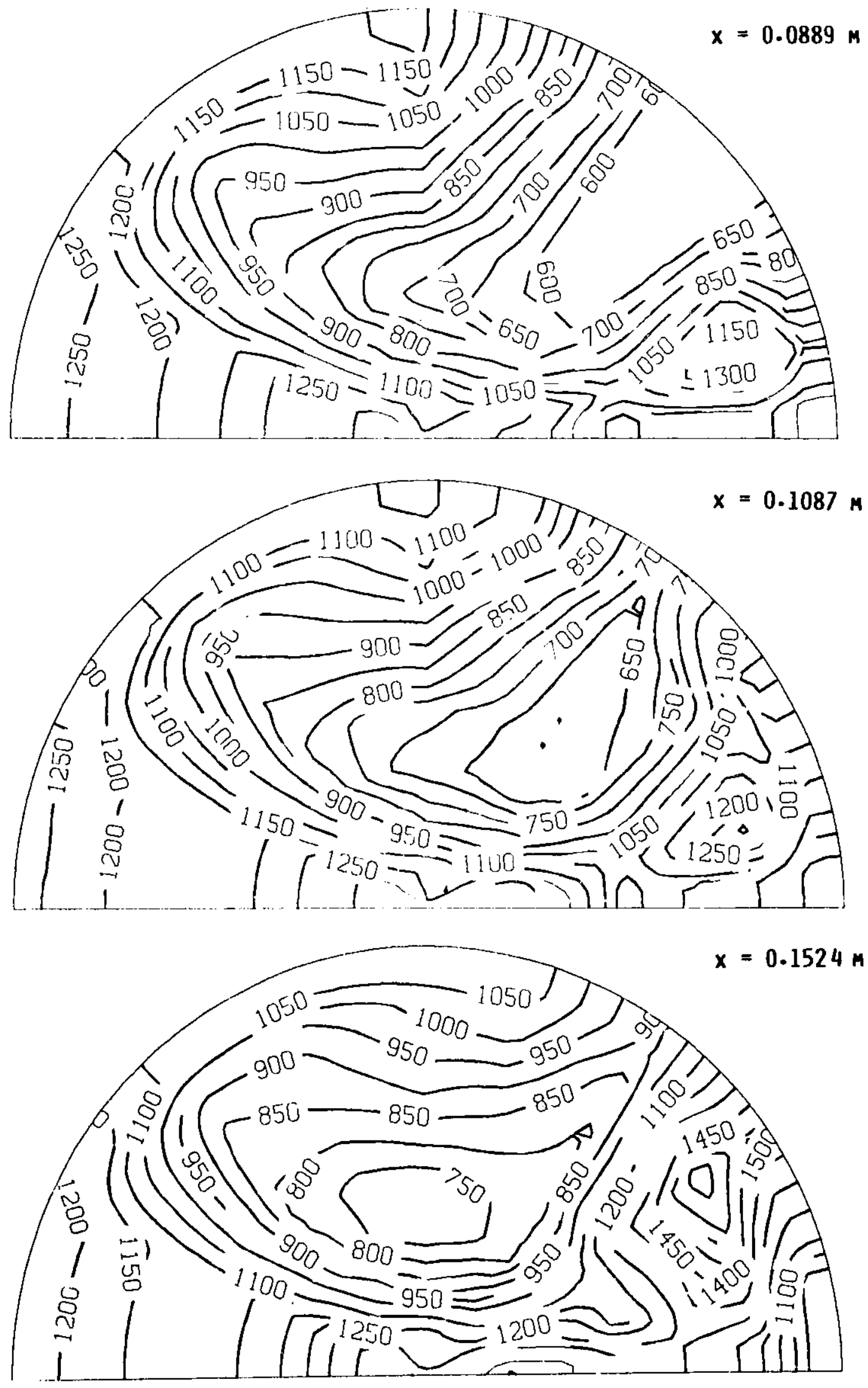

Figure 9. Cross-stream Temperature Distributions for $0.0 \mathrm{~m}$ Dome Helght 


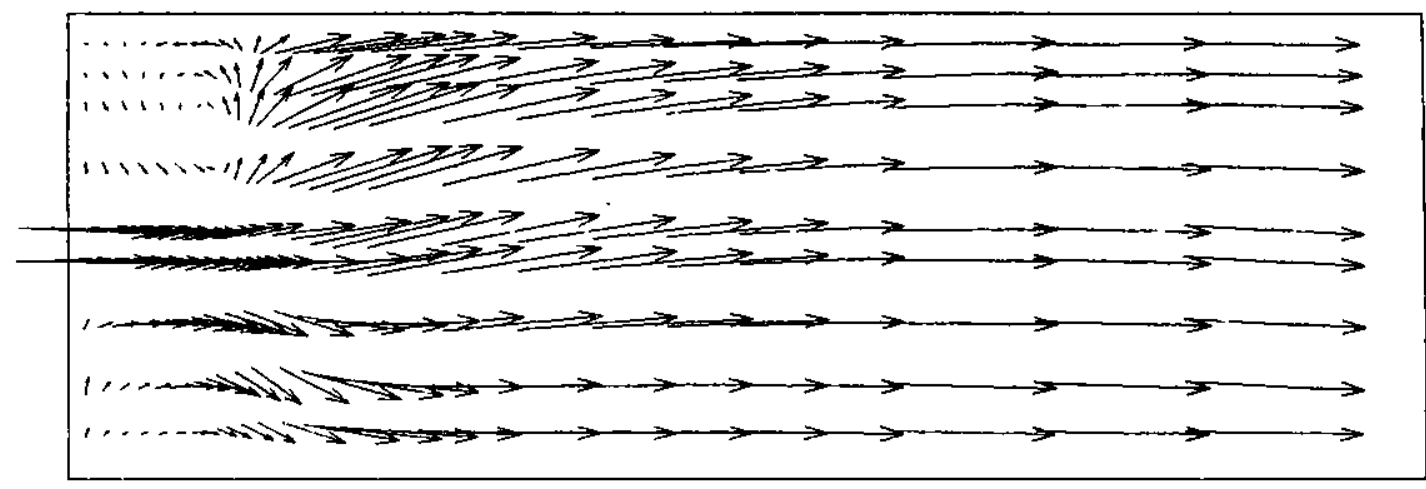

$\theta=5^{\circ}$

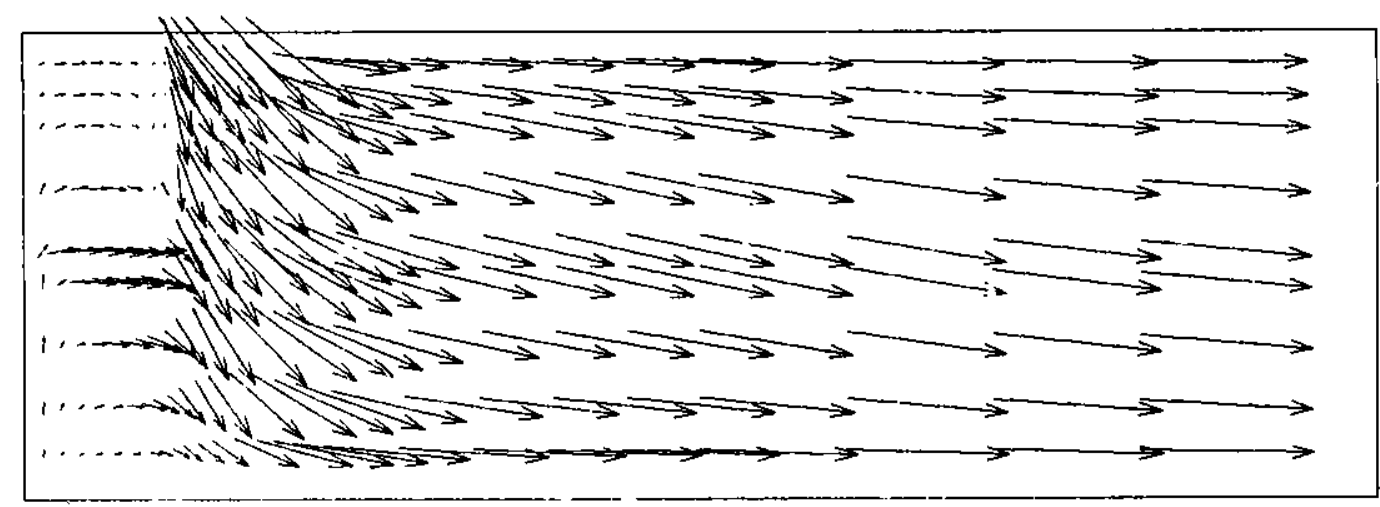

$\theta=35^{\circ}$

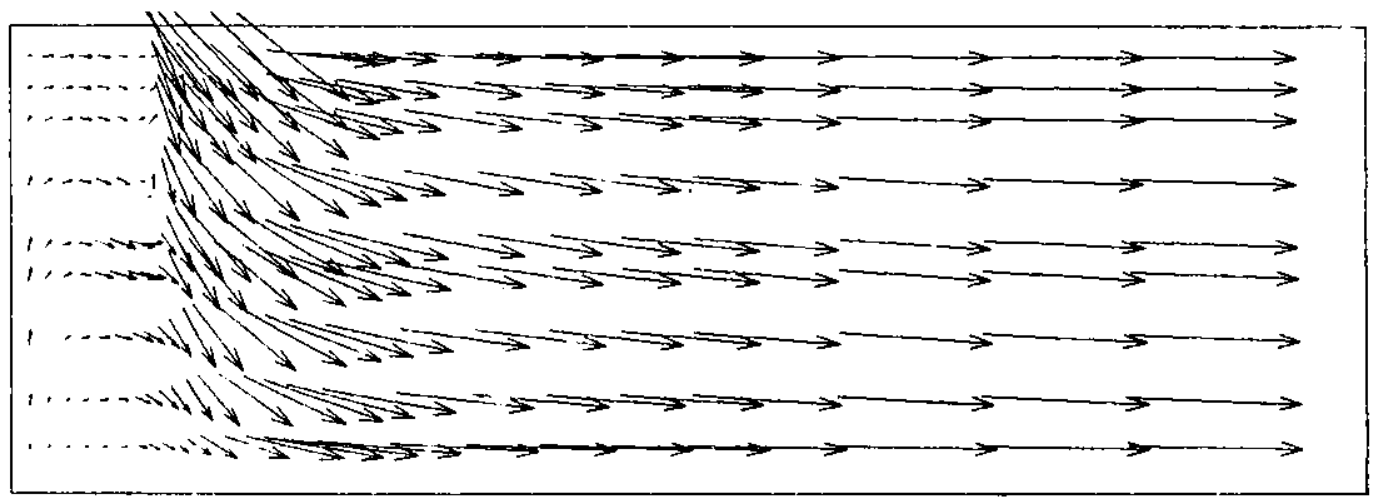

$\theta=54^{\circ}$

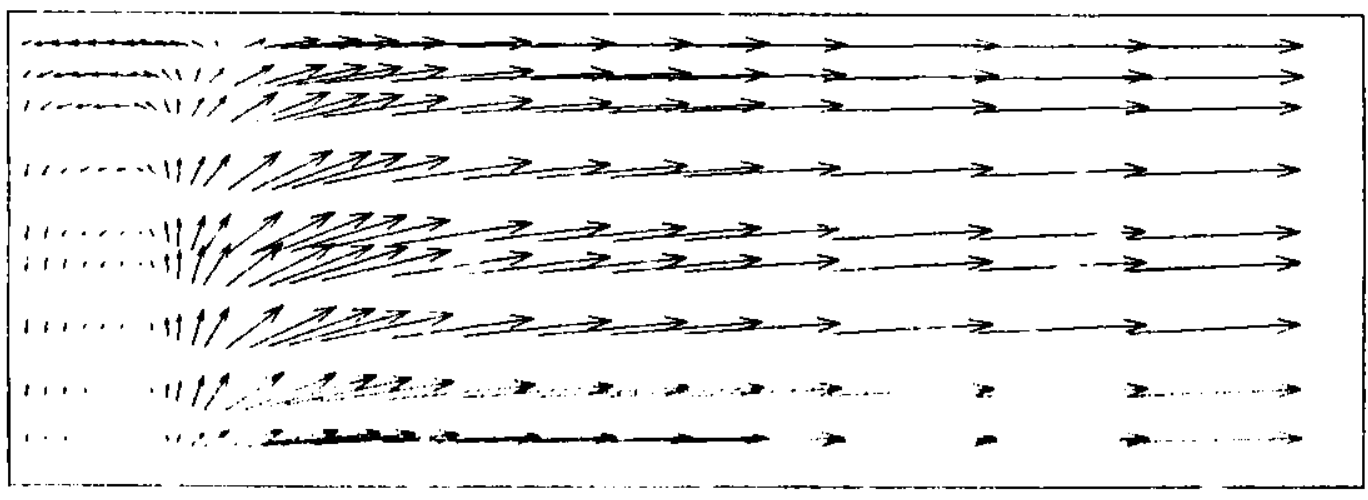




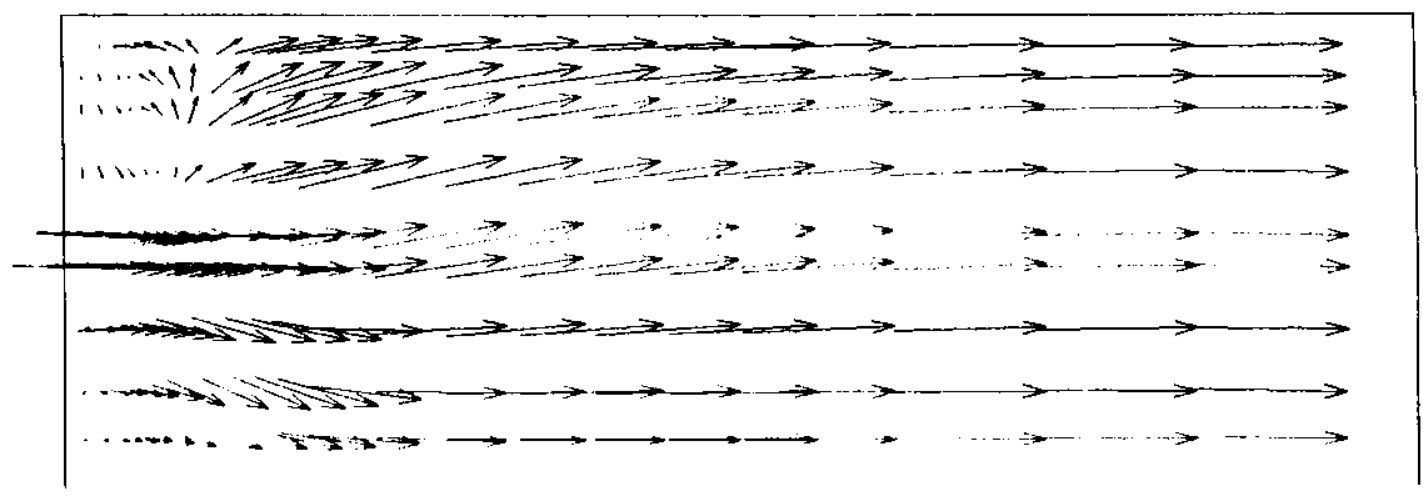

$\theta=5^{\circ}$
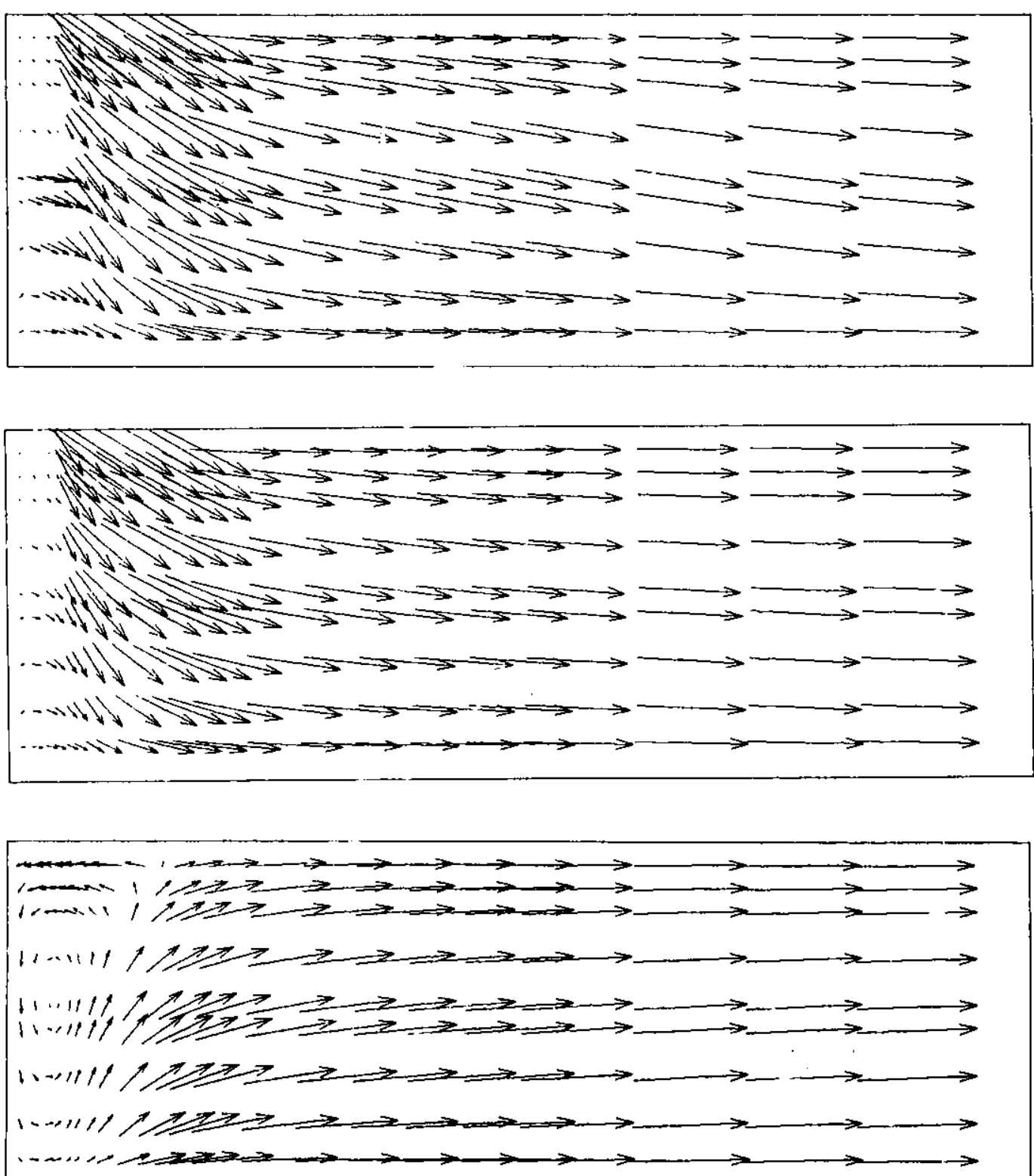

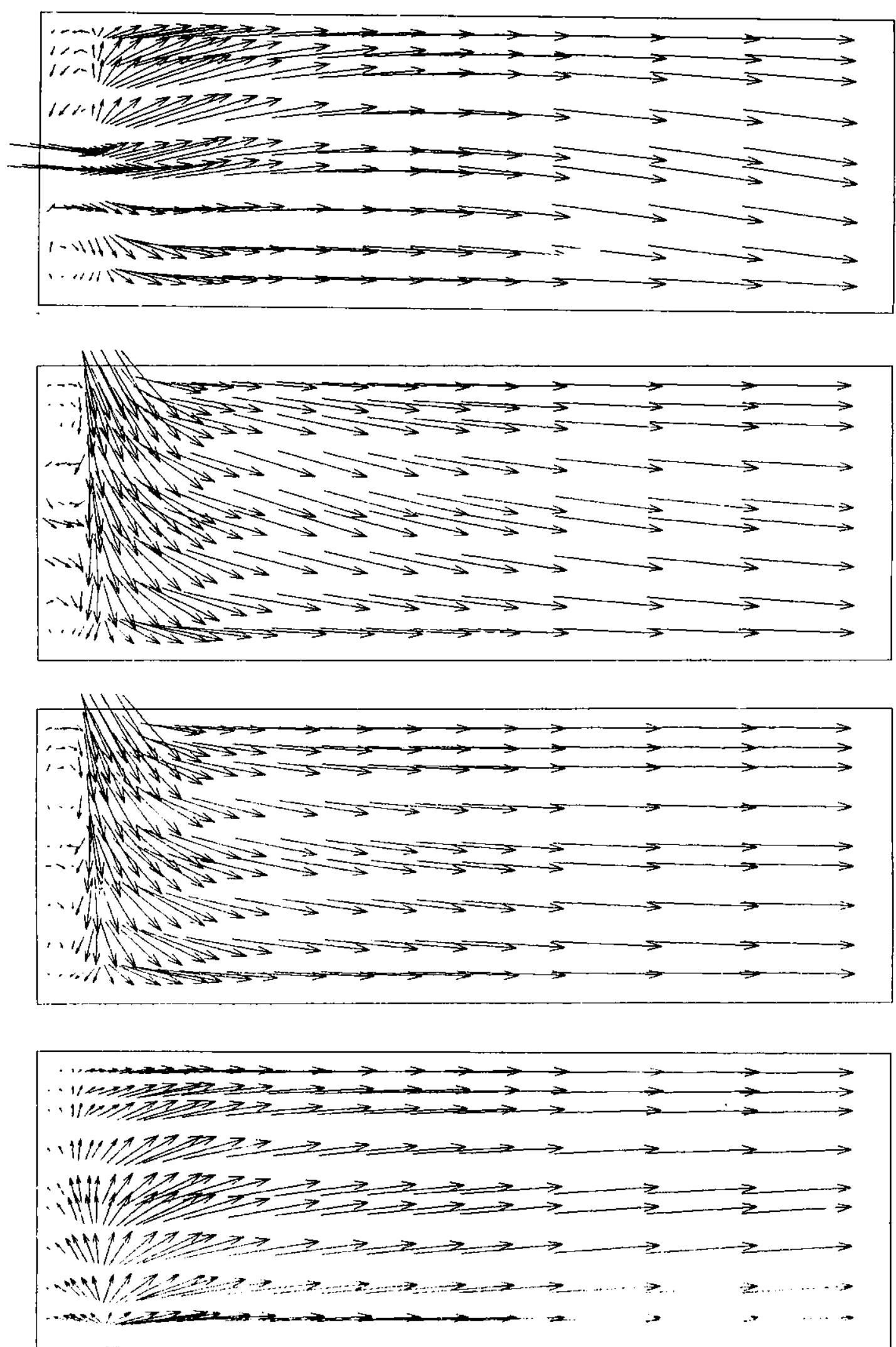


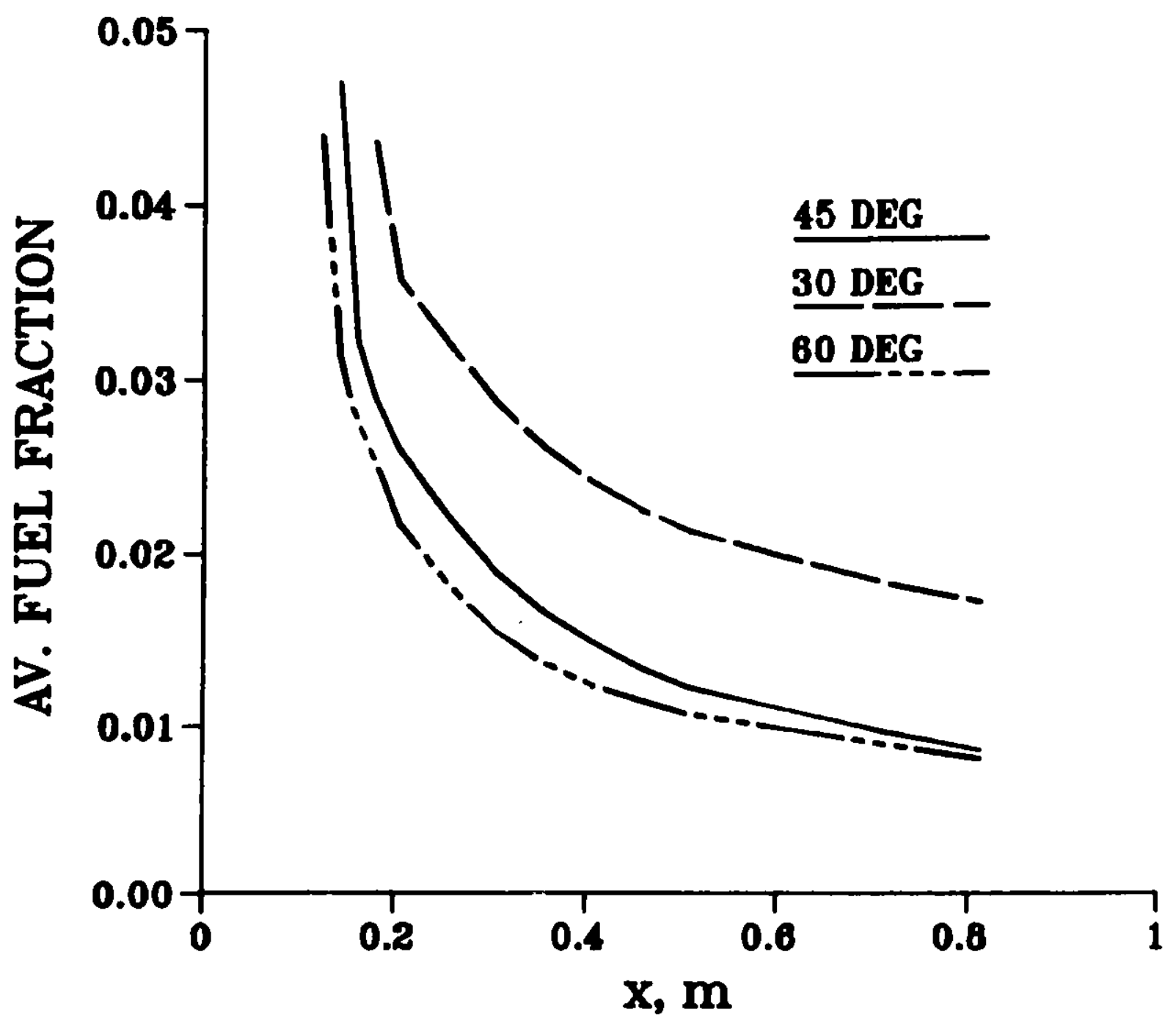

Figure 13. Axial Variation of Average Fuel Fraction for Different Side-arm Angles 


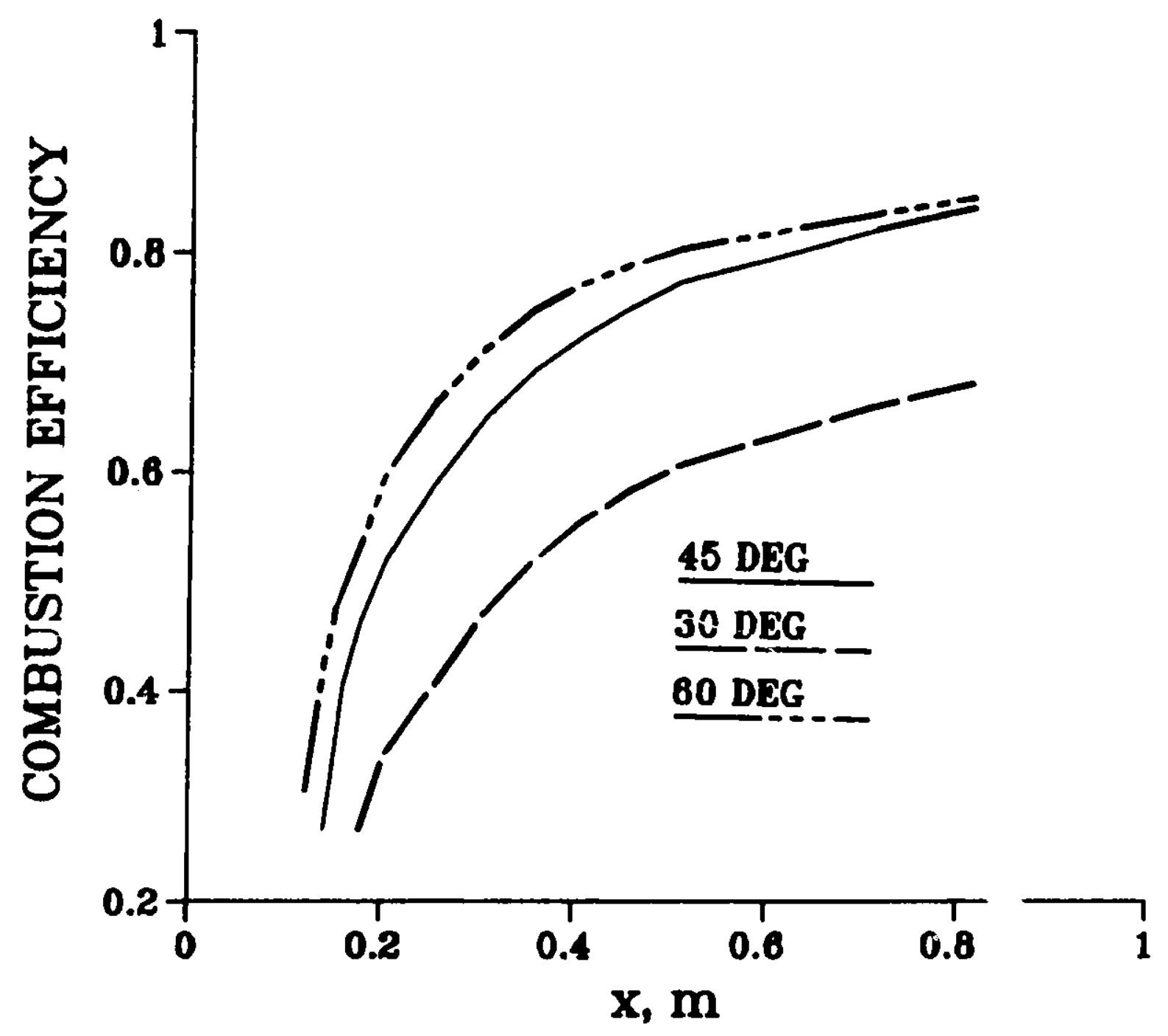

Figure 14. Axlal Variation of Average Combustion Efficiency for Different Side-arm Angles 

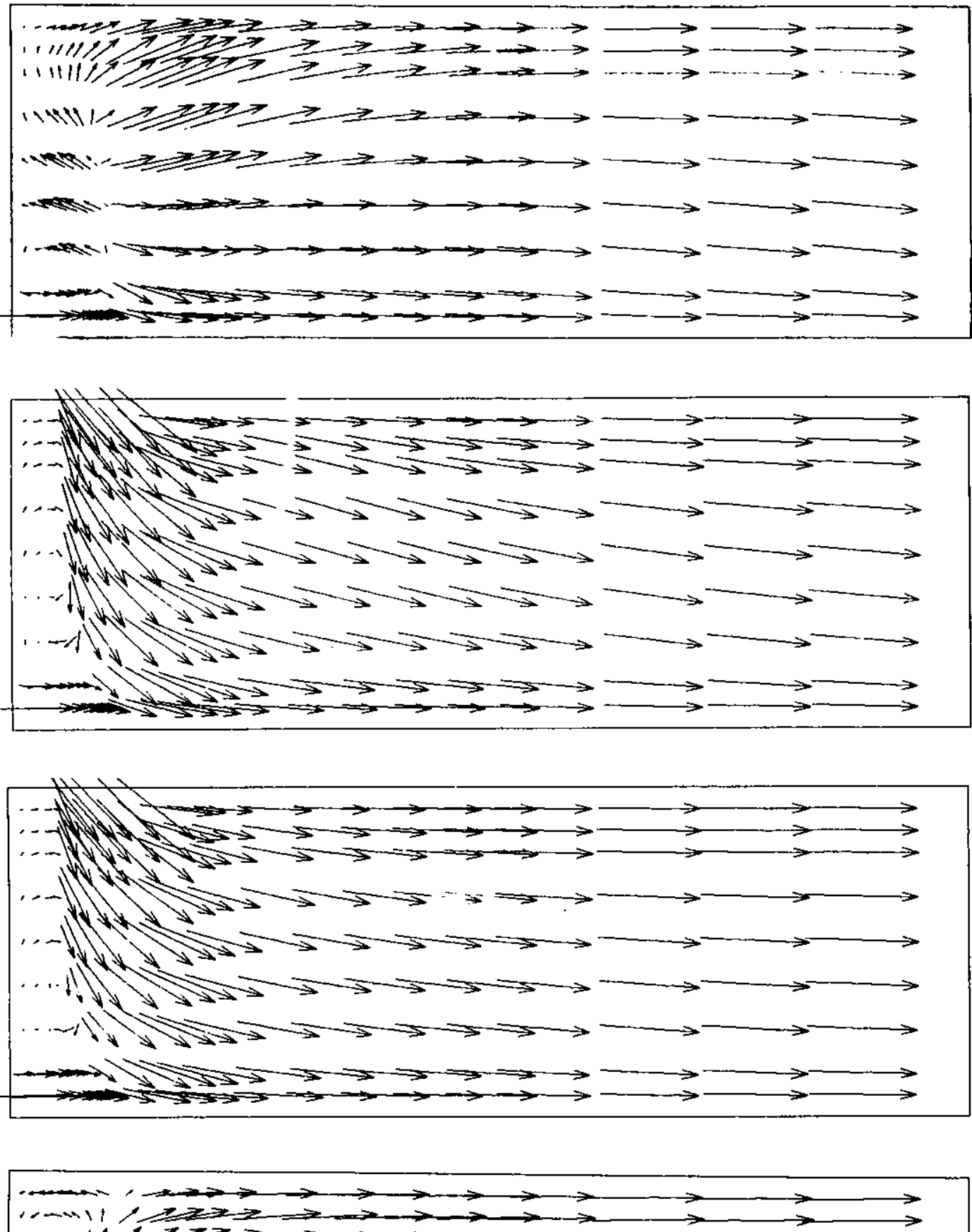

…

$1+\cdots \| 1 /, \geqslant \geqslant \geqslant \geq \longrightarrow$

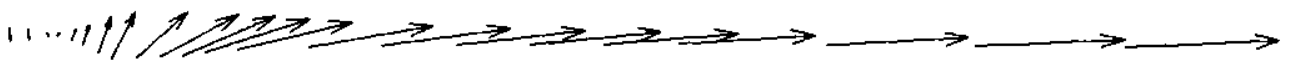

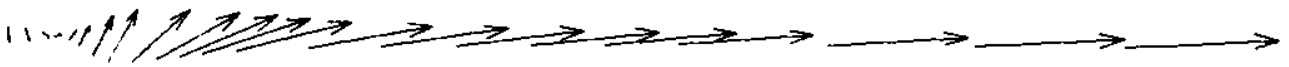

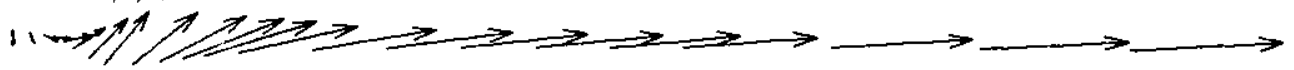

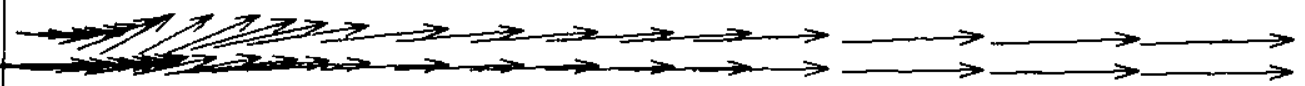

$\overrightarrow{150.00} \mathrm{M} / \mathrm{S}$

F1gure 15. Flow Patterns in Selected Azimuthal Planes for Concentric Fuel Injection 

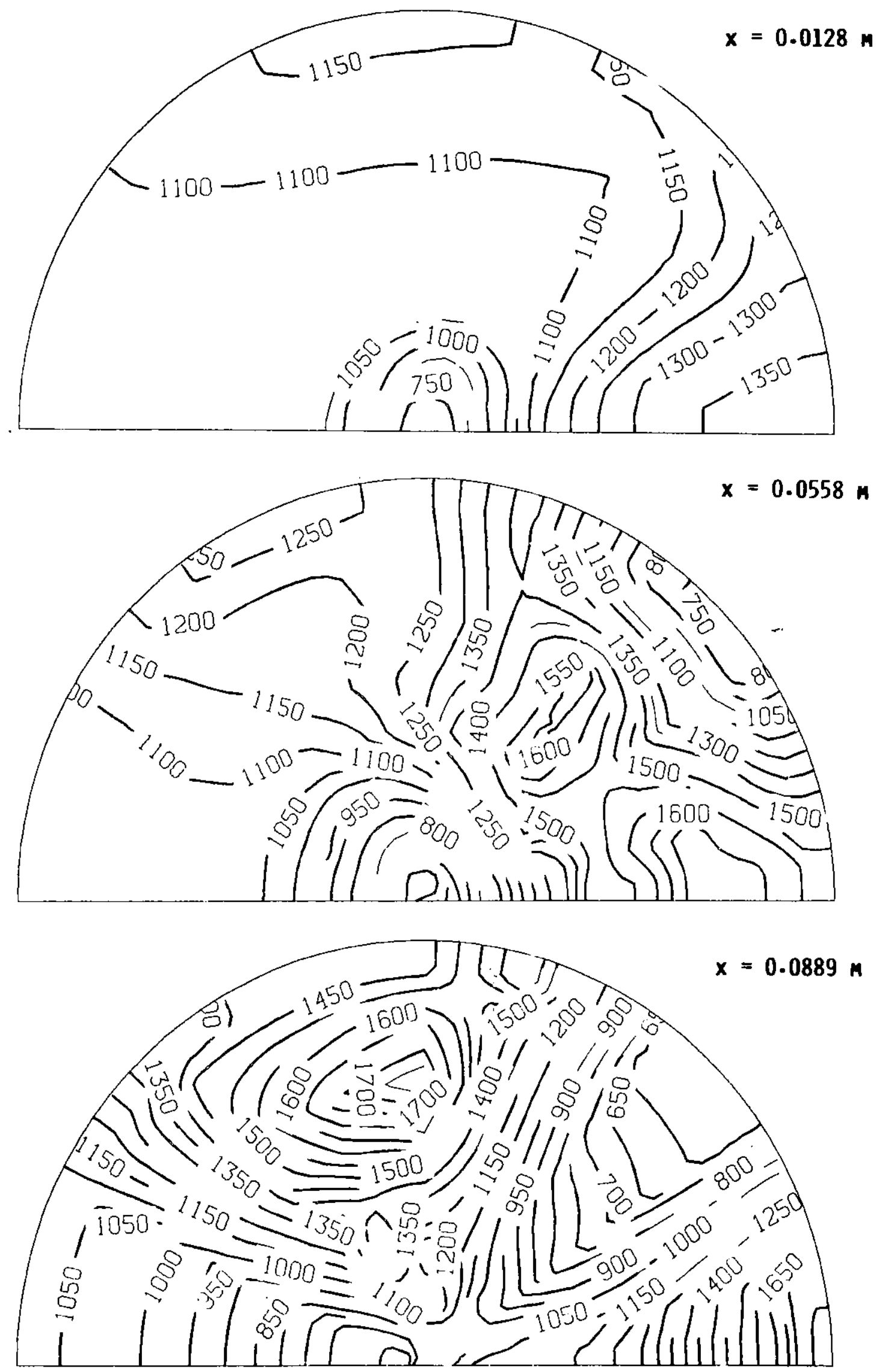

Figure 16. Cross-stream Temperature Distributions for Concentric Fuel Injection 

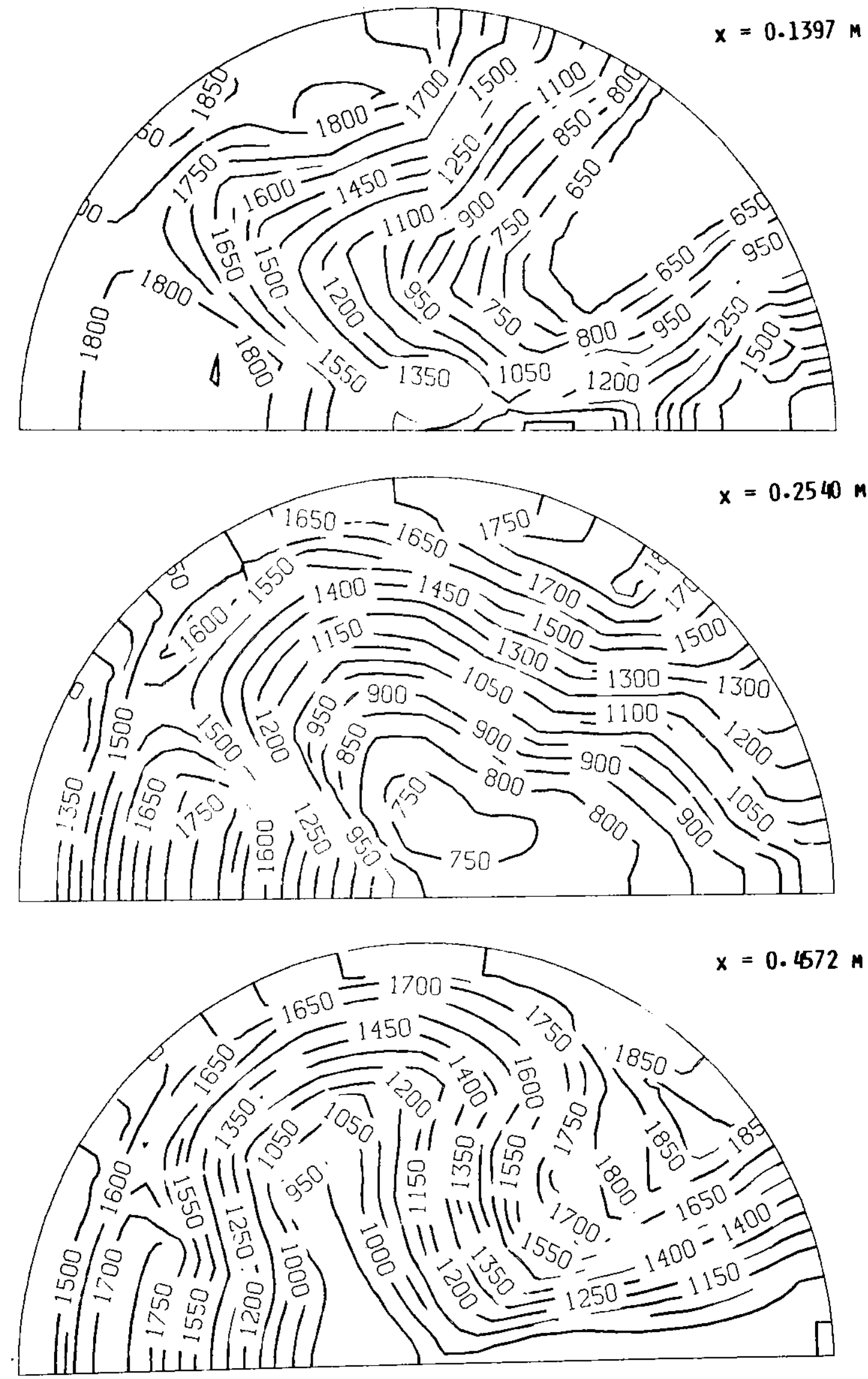

Figure 16. (contd) 


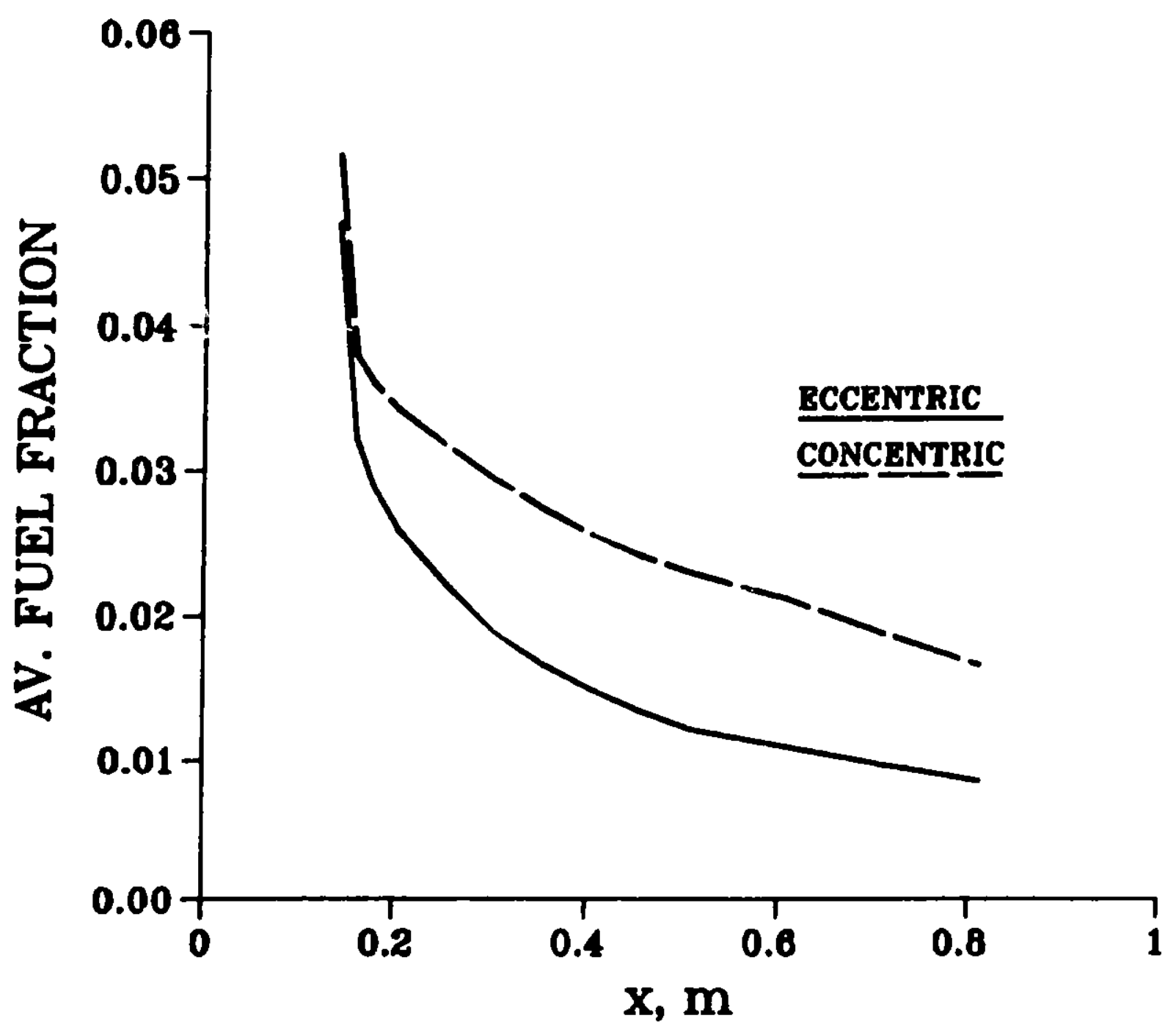

Figure 17. Axial Variation of Average Fuel Fraction for Concentric Fuel Injection 


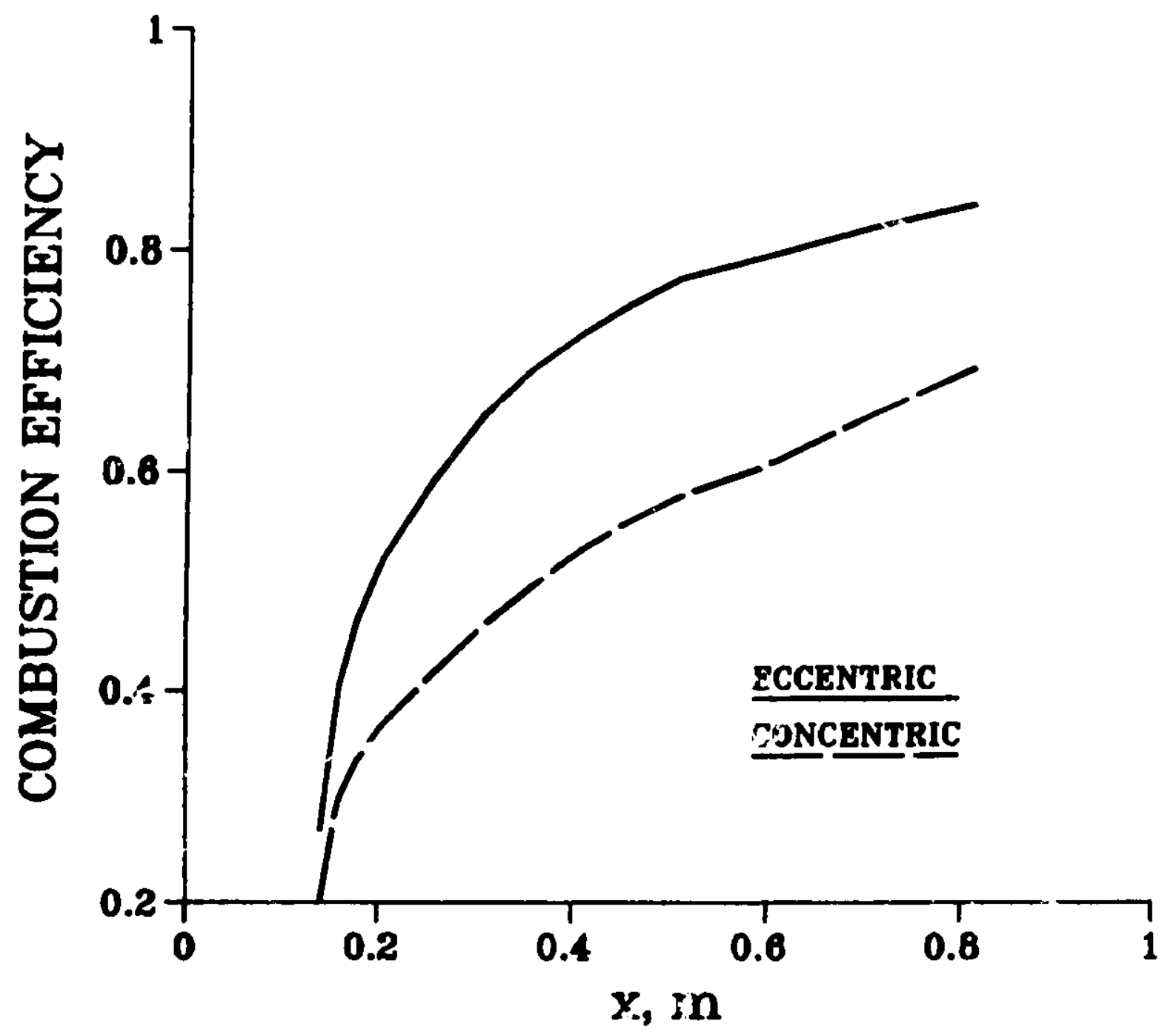

Figure 18. Axial Varlation of Average Combustion Efficiency for Concentric Fue1 Injection 
40

Table 1. Exchange Coefficients and Additional Source Terms

\begin{tabular}{lll}
\hline$\phi$ & $\Gamma_{\phi}$ & $s_{\phi}$ \\
\hline 1 & 0 & 0
\end{tabular}

$$
\begin{aligned}
u \quad \mu_{\ell}+\mu_{t} \quad \frac{\partial}{\partial x}\left(\mu_{\text {eff }} \frac{\partial u}{\partial x}\right)+\frac{1}{r} \frac{\partial}{\partial r}\left(r \mu_{\text {eff }} \frac{\partial v}{\partial x}\right)+\frac{1}{r} \frac{\partial}{\partial \theta}\left(\mu_{\text {eff }} \frac{\partial w}{\partial x}\right) \\
v \quad \mu_{\ell}+\mu_{t} \quad \frac{\rho w}{r}+\frac{\partial}{\partial x}\left(\mu_{\text {eff }} \frac{\partial u}{\partial r}\right)+\frac{1}{r} \frac{\partial}{\partial r}\left(r \mu_{\text {eff }} \frac{\partial v}{\partial r}\right) \\
+\frac{1}{r} \frac{\partial}{\partial \theta}\left(\mu_{\text {eff }} r \frac{\partial}{\partial r}(w / r)\right)-2 \frac{\mu_{\text {eff }}}{r}\left(\frac{\partial w}{r \partial \theta}+\frac{v}{r}\right)
\end{aligned}
$$

$$
\begin{aligned}
& w \quad \mu_{\ell}+\mu_{t} \quad-\frac{\rho v w}{r}+\frac{\partial}{\partial x}\left(\mu_{\text {eff }} \frac{\partial u}{r \partial \theta}\right)+\frac{\mu_{\text {eff }}}{r}\left(r \frac{\partial(w / r)}{\partial r}+\frac{1}{r} \frac{\partial v}{\partial \theta}\right) \\
& +\frac{1}{r} \frac{\partial}{\partial \theta}\left(\mu_{\text {eff }}\left(\frac{\partial w}{r \partial \theta}+\frac{2 v}{r}\right)\right)+\frac{1}{r} \frac{\partial}{\partial r}\left(r \mu_{\text {eff }}\left(\frac{1}{r} \frac{\partial v}{\partial \theta}-\frac{w}{r}\right)\right) \\
& \text { k } \quad \mu_{\ell}+\mu_{t} / \sigma_{k} \quad 0 ; P_{t}=\mu\left\{2\left(\frac{\partial u}{\partial x}\right)^{2}+2\left(\frac{\partial v}{\partial r}\right)^{2}+2\left(\frac{\partial w}{r \partial \theta}+\frac{v}{r}\right)^{2}\right. \\
& \left.+\left(\frac{\partial u}{\partial r}+\frac{\partial v}{\partial x}\right)^{2}+\left(\frac{\partial w}{\partial x}+\frac{\partial u}{r \partial \theta}\right)^{2}+\left(\frac{\partial v}{r \partial \theta}+\frac{\partial w}{\partial r}-\frac{w}{r}\right)^{2}\right) \\
& \varepsilon \quad \mu_{\ell}+\mu_{t} / \sigma_{\varepsilon} \\
& 0 \\
& \text { f } \quad \mu_{\ell}+\mu_{t} / \sigma_{f} \\
& 0 \\
& g \quad \mu_{\ell}+\mu_{t} / \sigma_{g} \quad 0 ; P_{g}=\mu_{t}\left\{\left(\frac{\partial f}{\partial x}\right)^{2}+\left(\frac{\partial f}{\partial r}\right)^{2}+\left(\frac{\partial f}{r \partial \theta}\right)^{2}\right\}
\end{aligned}
$$


Table 2. Puel Properties Used*

\begin{tabular}{|c|c|c|c|}
\hline Stolchiometric ratio (1) & \multicolumn{2}{|c|}{15.58} & \\
\hline Heat of reaction $\left(\mathrm{H}_{\mathrm{fu}_{u}}\right)$ & \multicolumn{2}{|c|}{$4.895 \times 10^{4} \mathrm{~kJ} / \mathrm{kg}$} & \\
\hline \multicolumn{4}{|l|}{ Molecular welghts: } \\
\hline Alr & \multicolumn{2}{|c|}{25.36} & \\
\hline $\mathrm{C}_{2} \mathrm{H}_{4}$ & \multicolumn{2}{|c|}{28.0} & \\
\hline Products & \multicolumn{2}{|c|}{25.55} & \\
\hline ipecif $1 \mathrm{c}$ heat constants $(\mathrm{kJ} / \mathrm{d} \in \mathrm{g} / \mathrm{kg})$ : & $a_{1}$ & $b_{1}$ & $c_{1}$ \\
\hline Atr & 0.927 & $2.580 \times 10^{-3}$ & $3.820 \times 10^{-8}$ \\
\hline Fue 1 & 0.404 & $4.360 \times 10^{-3}$ & $-1.353 \times 10^{-6}$ \\
\hline Products & 0.918 & $8.415 \times 10^{-4}$ & $-2.12 \times 10^{-7}$ \\
\hline
\end{tabular}

* The fuel properties correspond to those of gaseous ethylene $\left(\mathrm{C}_{2} \mathrm{H}_{4}\right)$.

\section{Table 3. Conditions for Base Caiculations}

$\begin{array}{ll}\text { Diameter of combustor } & 0.1524 \mathrm{~m}(6 \mathrm{In} .) \\ \text { Length of combustor } & 0.8636 \mathrm{~m}(34 \mathrm{ln} .) \\ \text { Dome height } & 0.0508 \mathrm{~m}(2 \mathrm{In.}) \\ \text { Angle of side arms } & 45^{\circ} \\ \text { Temperature of inlet air } & 556 \mathrm{~K}\left(1000^{\circ} \mathrm{R}\right) \\ \text { Afr flow rate (both arms) } & 1.814 \mathrm{~kg} / \mathrm{s}(4 \mathrm{lb} / \mathrm{s}) \\ \text { Fuel flow rate (F/A }=0.06) & 0.1088 \mathrm{~kg} / \mathrm{s}\left(0.241^{\mathrm{T} / \mathrm{s}}\right)\end{array}$




\section{ACKNONLEDGENTS}

This work was performed at Argonne National Laboratory under sponsorship of the Ramjet Technology Division, Wright Patterson Alr Force Base, through an interagency agreement.

\section{REFERENCES}

1. G. D. Streby, "Multi-ducted Inlet Combustor Research and Development," AFWAL-TR-83-2081, Aero Propulsion Laboratory, Air Force Wright Aeronautical Laboratories, WPAF3, 1983.

2. F. D. Stu11, R. R. Craig, G. D. Streby, and S. P. Vanka, "Investigation of a Dual Inlet Side Dump Combustor Using Liquid Fue1 Injection," AIAA-83-0420, 21st Aerospace Sclences Meeting, Reno, Nevada, 1983.

3. S. P. Vanka, F. D. Stull and R. R. Craig, "Analytical Characterfzation of Flow Fields in Side Inlet Dump Combustors," AIAA-83-1399, 19th Joint Propulsion Meeting, Seattle, WA, 1983.

4. R. R. Cralg, Unpublished Work, July 1984.

5. L. Chen and C. C. Tao, "Study of Side-Inlet Dump Combustor of Solid Ducted Rocket with Reacting Flow," AIAA-84-1378, AIAA/SAE/ASME 20th Jolnt Propulsion Conference, 1984.

6. B. E. Launder and D. B. Spalding, "The Numerical Computation of Turbulent Flows," Computer Methods in Applied Mechantcs and Englneering, Vol. 3, pp. 269-289, 1974.

7. D. B. Spalding, "Concentration Fluctuations in a Round Turbulent Free Jet," Chemical Engineering Sclence, Vol. 26, pp. 95-107, 1971.

8. S. E. Elgobash1 and W. M. Pun, "Concentration Fluctuations in Isothermal Turbulent Confined Coaxial Jets," Chemical Engineering Science, Vol. 32, pp. 161-166, 1977.

9. S. V. Patankar and D. B. Spalding, "A Calculation Procedure for Heat, Mass and Momentum Transfer in Three-Dimensional Parabolic Flows," International Journal of Heat Mass Transfer, Vo1. 15, pp. 1787-1806, 1972.

10. S. P. Vanka, "Computations of Turbulent Recirculating Flows with Fully Coupled Solution of Momentum and Continuity Equations," Argonne Natioual Laboratory Report, ANL-83-74, 1983. 


\section{Distribution for ANL-84-62}

Interna1:

R. S. Zeno

G. S. Rosenberg

P. R. Huebotter

C. A. Kot

M. Wambsganss

S. P. Vanka (50)

Y. W. Shin

R. E. Ho1tz

G. Leaf
N. Sather

M. Petrick

K. E. Kasza

S. K. Zussman

ANL Patent Dept.

ANL LIbrary

ANL Contract File

TIS F $¥$ les (6)

\section{External:}

DOE-TIC (27)

Manager, Chicago Operations of $f 1 c e, D O E$

E. T. Curran, Wright Patterson AFB, Oh1o

W. Beecroft, Wright Patterson AFB, Ohio

F. D. Stul1, Wright Patterson AFB, Ohto

R. R. Craig, Wright Patterson AFB, Oh1o

R. S. Boray, Wright Patterson AFB, Ohio

H. H. Chiu, Mech. Engg. Dept., University of Illinols, Chicago

Components Technology Division Review Committee:

P. Alexander, Flopetrol Johnston Schlumberger, Houston, Texas

D. J. Anthony, General Electric Co., San Jose

A. A. Bishop, U. Pittsburgh

B. A. Boley, Northwestern U.

F. W. Buckman, Delian Corp., Monroeville, Pa.

R. Cohen, Purdue U.

J. Weisman, U. Cincinnati 